\title{
Privacy-preserving Fusion of IoT and Big Data for E-health
}

\author{
Yang Yang ${ }^{\mathrm{a}, \mathrm{b}, \mathrm{c}, \mathrm{d}}$, Xianghan Zheng, ${ }^{\mathrm{a}, \mathrm{c}, \mathrm{d}}$, Wenzhong Guo ${ }^{\mathrm{a}, \mathrm{c}, \mathrm{d}}$, Ximeng Liu ${ }^{\mathrm{a}, \mathrm{b}}$, \\ Victor Chang ${ }^{\mathrm{e}}$ \\ ${ }^{a}$ College of Mathematics and Computer Science, Fuzhou University, Fuzhou, China. \\ ${ }^{b}$ University Key Laboratory of Information Security of Network Systems (Fuzhou \\ University), Fujian Province, China. \\ ${ }^{c}$ Fujian Provincial Key Laboratory of Network Computing and Intelligent Information \\ Processing, Fuzhou University, Fuzhou, China \\ ${ }^{d}$ Key Laboratory of Spatial Data Mining \& Information Sharing, Ministry of Education, \\ Fuzhou, China \\ ${ }^{e} I B S S$, Xi'an Jiaotong-Liverpool University, Suzhou, China.
}

\begin{abstract}
In this paper, we propose a privacy-preserving e-health system, which is a fusion of Internet-of-things (IoT), big data and cloud storage. The medical IoT network monitors patient's physiological data, which are aggregated to electronic health record (EHR). The medical big data that contains a large amount of EHRs are outsourced to cloud platform. In the proposed system, the patien$\mathrm{t}$ distributes an IoT group key to the medical nodes in an authenticated way without interaction round. The IoT messages are encrypted using the IoT group key and transmitted to the patient, which can be batch authenticated by the patient. The encrypted EHRs are shared among patient and different data users in a fine-grained access control manner. A novel keyword match based policy update mechanism is designed to enable flexible access policy updating without privacy leakage. Extensive comparison and simulation results demonstrate that the algorithms in the proposed system are efficient. Comprehensive analysis is provided to prove its security.
\end{abstract}

Email addresses: yang.yang.research@gmail.com (Yang Yang), xianghan.zheng@fu.edu.cn (Xianghan Zheng), guowenzhong@fzu.edu.cn (Wenzhong Guo), snbnix@gmail.com (Ximeng Liu), victorchang.research@gmail.com (Victor Chang)

Preprint submitted to Future Generation Computer Systems

December 13, 2017 
Keywords: Internet-of-things, big data, privacy-preserving, cloud storage, access control, keyword match based policy update

\section{Introduction}

The rapid development of Internet of Things (IoT) 1, 2] has greatly changed our daily life, especially in the electronic health (e-health) domain [3]. The patients with chronic diseases or severe illnesses are equipped with implanted or on the surface medical sensors to monitor various kinds of physiological data. These medical nodes are accommodated in the e-health IoT network, and the collected physiological data are gathered to patient's gateway device through Internet, which forms patient's electronic health record (EHR). As the data accumulated, the EHRs of the patients become medical big data with high volume, which faces a lot of challenges, such as the information privacy, search, updating and sharing. It is emergent to design a privacy-preserving e-health system for the fusion of IoT and medical big data [4, 5, 6, which handles these challenges.

In the e-health IoT network, the collected physiological data may leak patient's privacy and should to be encrypted to guarantee the confidentiality. The key distribution in IoT is a major issue to be handled such that the patient's gateway device and all the medical nodes share the same IoT symmetric key. The existing schemes requires interaction between the gateway device and the medical nodes to negotiate a secret key, which consumes a large number of transmission and computation costs. Considering the low battery supply and weak computing capability of the tiny medical sensors, it is desirable to generate the IoT group key (among patient and the medical nodes) without key negotiation rounds and at the same ensure the security, such as the source authentication. After the IoT key is distributed, the medical nodes encrypt the health data using the IoT key and transmit them to the patient. To resist impersonation attack, the patient authenticates these IoT ciphertexts to make sure that they are sent by the nodes in his own IoT network. Batch verification method should 
be designed to accelerate the authenticating speed.

The EHRs in the e-health big data system also requires the privacy protection, and to be shared between patients and the designated data users. Attribute based encryption $(\mathrm{ABE})$ provides an ideal method to realize fine-grained access control and can be adopted in the medical big data system. The system users are assigned with attribute secret key and the patients' EHRs are encrypted with access policy such that only the users with proper attributes secret key are authorized to access to patients' encrypted EHRs.

The policy updating is a big challenge in health big data environment because the encrypted EHRs are outsourced to the medical cloud rather than locally stored. It is not realistic for the patient to download all the EHR ciphertexts with an old access policy, decrypt and re-encrypt them with a new access policy, which brings heavy transmission and computation burden to the patient. On the other hand, the patients hope to make the access policy updating in a finer controlled manner such that only the encrypted EHRs contain certain keywords are updated to the new access policy. This problem is not considered and solved in the existing works.

\subsection{Our Contributions}

To handle the above challenges, a privacy-preserving e-health system with the fusion of IoT and big data is designed and the main contributions are summarized below.

- Anonymity and traceability of patient and medical node: In the $\mathrm{e}-$ health system, the identities of patient and the medical nodes in patient's IoT network are sensitive and may leak the privacy. In this system, anonymous identities are assigned for both patient and medical nodes, which is calculated from their real identities. If an anonymous patient is found dishonest or misbehaving, the trusted authority is capable to trace his real identity. If an anonymous medical node is compromised and utilized to launch attack in patient's IoT network, the patient can also recover the node's real identity. 
- Authenticated IoT key distribution: In order to guarantee the confidentiality of the messages transmitted in the health IoT network, the patient generates a symmetric key and sends it to all the medical nodes (in patient's IoT network) in a privacy-preserving way. A key extraction auxiliary message is created by the patient to encapsule the IoT key. Receiving the message, the medical nodes authenticate that whether the auxiliary message is indeed sent by the patient to prevent impersonation attack.

- Authenticated IoT ciphertext transmission: After the IoT key is extracted by the medical nodes, the generated IoT messages are encrypted by the key and sends to the patient. To ensure the reliability of the source, the patient authenticates the IoT ciphertext and then decrypt it. A large amount of IoT ciphertexts may arrive at the same time period, our system also provides a batch verification algorithm to improve the efficiency.

- Lightweight fine-grained access control: The e-health big data are encrypted and stored in a cloud platform. To prevent unauthorized data access, our system designs an expressive and lightweight fine-grained access control mechanism. The patient controls the electronic health record (EHR) encryption procedure, and defines an access policy such that the data users with specific attributes can decrypt patient's medical files. The algorithms in the access control mechanism are lightweight constructions.

- Flexible subset keyword match based access policy update: We design a novel access policy update mechanism based on keyword match. When the patient wants to change the access policy, he does not need to download all his EHR ciphertexts, decrypt them and re-encrypt them with a new access policy. In our system, the patient generates a privacypreserving policy update query, which is sent to the cloud server. Then, the cloud server updates the match files without knowing the plaintext. A highlight is that the policy update process is keyword match based. If the patient wants to update the access policy of the EHRs that contains certain 
keywords, the query keywords are embedded in the policy update query.

Only the access policy match files that contains these query keywords as subset keywords will be updated.

\subsection{Related Work}

\subsubsection{Security of IoT and Big Data}

The Internet-of-things (IoT) network contains a lot of security and privacy concerns. Arias et al. [7] analyze the hardware security of the wearable devices and user privacy in wearable IoT system. The secure storage and forward proxy problem is studied in [8] for the dynamic machine-to-machine IoT application. Mutual authentication protocol is designed in 9 for smart city IoT application, which is constructed based on learning with errors complexity assumption. Zhang et al. 10] suggest a three-factor key agreement protocol for e-health system, which supports dynamic authentication. Another key agreement protocol for multi-gateway IoT network is proposed in [11. The public auditing and verifiable updating issues are investigated in [12. The big data security in smart grid system is considered in [13. Liu et al. [14] design a merkel hash tree to realize public auditing in the big data storage system. A secure deduplication scheme [15] is constructed for encrypted big data.

\subsubsection{Searchable Encryption}

Searchable encryption is a technology to realize keyword search functor over encrypted data. In 2004, Boneh et al. 16 propose a searchable encryption in the public key setting. Xu et al. [17] study the fuzzy keyword search and construct a concrete probable security scheme. The searchable proxy re-encryption is investigated in 24] to resist post-quantum attack. Wang et al. 19] propose a ranked keyword search scheme over outsourced cloud data, and Cao et al. [20] construct a rank scheme supporting multiple keywords. A dynamic searchable encryption system is suggested by Cash et al. [21] for very-large database. Li et al. 32 studies the quality of protection (QoP) and quality of experience (QoE) issues of searchable encryption in the mobile cloud application. Yang et 
al. 23] investigate the flexible conjunctive keyword search and time controlled authorization problems and construct a concrete searchable encryption system. Some other research work 24 is proposed to realize flexible keyword search.

\subsubsection{Attribute Based Encryption}

The concept of attribute-based encryption (ABE) is introduced by Goyal et al. 25] and has received extensive extension [26, 27, 28, since then. Yang et al. propose the sharable and traceable ABE scheme in [29] and study the break-glass access in emergency scenario in [30. The outsourced policy update problem is investigated in [31, where different types of access policies are taken into consideration. To update linear secret sharing scheme (LSSS) based access policy, the scheme in 31. designs a policy compare algorithm to divide the update policy into three types and disparate methods to update the old policy according the different types. The policy updating method in [31] is utilized to construct other ABE schemes in [32, 33. The searchable encryption and ABE are integrated in [34, 35] such that the authorized data user (with attributes satisfy the access policy) can utilize a keyword based trapdoor to retrieve on data owner's encrypted files. The dynamic auditing and attribute revocation issues are studied in [36].

\subsection{Road Map}

The rest of this paper is organized as follows. In Section 2, bilinear paring, hardness assumption and linear secret sharing scheme are introduced. Section 3 describes the system model and security model. In Section 4, we propose the system to fuse the health IoT network and big data storage platform. In Section 5 and 6 , the security of the proposed system is analyzed. In Section 7, a comprehensive performance analysis and comparison is given. Section 8 concludes this paper. 


\section{Preliminary}

\subsection{Bilinear Pairing and Hardness Assumption}

Let $G$ and $G_{T}$ be cyclic groups and $g$ be a generator of $G$. The bilinear map $e: G \times G \rightarrow G_{T}$ has the following characteristics: (1) bilinear: $\forall u, v \in G$ and $\forall a, b \in \mathbb{Z}_{p}, e\left(u^{a}, v^{b}\right)=e(u, v)^{a b} ;(2)$ non-degenerate: $e(g, g) \neq 1$; (3) computable: $e$ can be efficiently calculated.

Decisional Bilinear Diffie-Hellman (DBDH) Assumption. Let $a, b, c \in$ $Z_{p}$ be chosen at random, and $g$ be a generator of $G$. An adversary $\mathcal{A}$ is given a tuple $\vec{y}=\left(g, g^{a}, g^{b}, g^{s}\right)$, it is hard for $\mathcal{A}$ to distinguish $e(g, g)^{a b s}$ from a random element $Z$ from $G_{T}$.

\subsection{Linear Secret Sharing Scheme}

Definition 1 (Linear Secret Sharing Scheme (LSSS) [37]). A secret-sharing scheme $\Pi$ over a set of parties $\mathcal{P}$ is called linear (over $Z_{p}$ ) if

- The shares for each party form a vector over $Z_{p}$.

- There exists a matrix $\mathbb{A}$ with $n_{1}$ rows and $n_{2}$ columns called the sharegenerating matrix for $\Pi$. For all $i=1, \cdots, n_{1}$, the $i$ th row $\mathbb{A}_{i}$ of the matrix $\mathbb{A}$ is labeled by a party $\rho(i)$ ( $\rho$ is a function from $\left\{1, \cdots, n_{1}\right\}$ to $\mathcal{P}$ ). Set the column vector $V^{\prime}=\left(s, v_{2}, \cdots, v_{n}\right)$, where $s \in Z_{p}$ is the secret to be shared and $v_{2}, \cdots, v_{n} \in Z_{p}$ are randomly chosen. $\mathbb{A} \cdot V^{\prime}$ is the vector of $n_{1}$ shares of the secret $s$ according to $\Pi$ and the share $\mathbb{A}_{i} \cdot V$ belongs to party $\rho(i)$.

Suppose that $\Pi$ is an LSSS for the access structure $\Phi$. Let $S \in \Phi$ be any authorized set and $I \subset\left\{1, \cdots, n_{1}\right\}$ be defined as $I=\{i: \rho(i) \in S\}$. Then, there exists constants $\left\{\lambda_{i} \in Z_{p}\right\}_{i \in I}$ such that, if $\left\{s_{i}\right\}_{i \in I}$ are valid shares of any secret $s$ according to $\Pi$, then $\sum_{i \in I} \lambda_{i} s_{i}=s$ and $\sum_{i \in I} \lambda_{i} \mathbb{A}_{i}=(1,0, \cdots, 0)$. Furthermore, it is shown in 37] that these constants $\left\{\lambda_{i}\right\}_{i \in I}$ can be found in time polynomial in the size of the share-generating matrix $\mathbb{A}$. For unauthorized sets, no such constants exist. 


\section{System and Security Model}

\subsection{System Model}

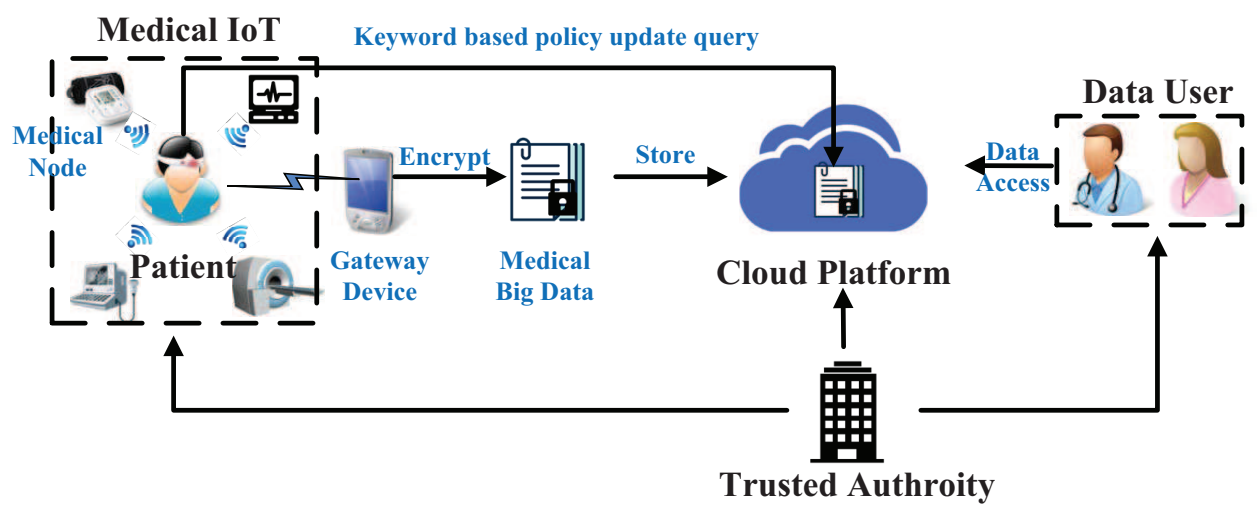

Figure 1: System Model

The system model for the fusion of IoT and big data in this work is depicted in Figure 1. The following roles are involved in the system.

- Trusted authority $(T A)$ : is fully trusted in the system, and responsible to generate the public parameter and master secret key. $T A$ is also tasked to create the public/secret key pair for the patients and data users.

- Patient $(P A)$ : is monitored by the medical IoT network, and responsible to generate the public/secret key pair for the medical nodes in the network. $P A$ also generates IoT key to ensure the privacy-preserving message transmission in its medical IoT network. The collected data from IoT are aggregated to electronic health record (EHR), which is encrypted by $P A$ with proper access policy such that only the designated set of users are authorized to decrypt the EHRs. PA can also utilizes the keyword match based access policy update mechanism to update the pre-defined access policy of the encrypted EHRs stored in the cloud server.

- Medical node $(M N)$ : in the patient's medical IoT network is responsible 
to collect the physiological data and encrypts it using the IoT key. Then, $M N$ sends the encrypted IoT message to patient.

- Data User $(U)$ : receives the attribute public/secret key from $T A$ and is able to decrypt patient's encrypted EHRs using the attribute secret key if $U$ 's attribute set satisfies the EHR's access policy.

- Cloud platform: is responsible to stored the encrypted EHRs. When the keyword match based access policy is received from the patient, the cloud platform runs ciphertext update algorithm to find the match EHRs ciphertexts and update the access policy.

\subsection{Security Model}

The privacy-preserving system for the fusion of IoT and big data is indistinguishable against chosen plaintext and chosen keyword attach (IND-CPCKA) if there is no probabilistic polynomial time adversary $\mathcal{A}$ can win the following interactive game (between adversary $\mathcal{A}$ and challenger $\mathcal{C}$ ) with non-negligible advantage.

- Setup: The global setup algorithm is executed and the public parameter $P P$ is generated, which is given to $\mathcal{A}$ and the master secret key $M S K$ is kept secret from $\mathcal{A}$.

- Phase 1. The following queries are issued by the adversary $\mathcal{A}$.

- Patient's secret key query: $\mathcal{A}$ queries on the patient PA's attribute secret key with attribute set $S=\left(a t t r_{1} \cdots a t t r_{n_{P A}}\right)$. $\mathcal{C}$ constructs $P A$ 's secret key $S K_{P A}$, which is sent to $\mathcal{A}$.

- Users's secret key query: $\mathcal{A}$ queries on the user $U$ 's attribute secret key with attribute set $S=\left(a t t r_{1} \cdots a t t r_{n_{U}}\right) . \mathcal{C}$ constructs $U$ 's secret key $S K_{U}$, which is sent to $\mathcal{A}$.

- Mobile node's secret key query: $\mathcal{A}$ queries on the mobile node $M N$ 's secret key, which belongs to the patient $P A$ 's IoT network. $\mathcal{C}$ constructs $M N$ 's secret key $S K_{M N}$, which is sent to $\mathcal{A}$. 
- Keyword match based policy update query: $\mathcal{A}$ queries on the patient $P A$, keyword set $Q W=\left(w_{\sigma_{1}}, \cdots, w_{\sigma_{l_{2}}}\right)$ and access policies $(\mathbb{A}, \rho)$, $\left(\mathbb{A}^{\prime}, \rho^{\prime}\right), \mathcal{C}$ constructs the policy update query $P U Q$, which is sent to $\mathcal{A}$.

- Challenge: $\mathcal{A}$ sends to $\mathcal{C}$ a challenge access policy $\left(\mathbb{A}^{*}, \rho^{*}\right)$, a challenge patient identity $P A^{*}$, a challenge EHR $M^{*}$, two EHR encryption keys $\left(\Upsilon_{0}^{*}, \Upsilon_{1}^{*}\right)$, and two challenge keyword sets $\left(K W_{0}^{*}, K W_{1}^{*}\right)$, where $\mathbb{A}^{*} \in$ $Z_{p}^{n_{1}^{*} \times n_{2}^{*}}$ and $\rho^{*}$ maps $\mathbb{A}^{*}$ s rows to attributes. It is required that the secret key of the attribute set $S$ that satisfies the challenge access policy $\left(\mathbb{A}^{*}, \rho^{*}\right)$ is not queried in phase 1 . Moreover, the secret key of $P A^{*}$ is not queried. $\mathcal{C}$ flips random coins $\mu_{1}, \mu_{2} \in\{0,1\}$ and the challenge EHR ciphertext $C T^{*}$ of $\left(\Upsilon_{\mu_{1}}^{*}, K W_{\mu_{2}}^{*}\right)$ is constructed, which is sent to $\mathcal{A}$.

- Phase 2: It is the same as in Phase 1 except that the secret key of the attribute set $S$ that satisfies the challenge access policy $\left(\mathbb{A}^{*}, \rho^{*}\right)$ is not allowed to be queried. In addition, the secret key of $P A^{*}$ is not allowed to be queried.

- Guess: Finally, $\mathcal{A}$ outputs $\mu_{1}^{\prime}, \mu_{2}^{\prime} \in\{0,1\}$. If $\mu_{1}^{\prime}=\mu_{1}$ and $\mu_{2}^{\prime}=\mu_{2}, \mathcal{A}$ wins the game.

\subsection{System Requirements}

This fusion of IoT and big data system needs to guarantee the following functional and security requirements.

- Patient anonymity and traceability: Patient's identity is sensitive in the e-health system and should be hidden from the other system users. The privacy-preserving system needs to provide patient anonymity function. On the other hand, patient anonymous identity should be traceable to prevent misbehave issues.

- User anonymity and traceability: The data users include the healthcare staffs, patients' friends and relatives etc. Their identity may leak patients 
identity and disease related information and needs to be protected by the anonymous mechanism. On the other hand, the anonymous identity should be traceable to prevent misbehave issues.

- Medical node anonymity and traceability: The real identity of medical nodes in IoT network may leak the variety of disease of the patient and should be protected by the anonymous mechanism. If a medical node is compromised and utilized to launch attacks, its real identity should be revealed by the trace mechanism.

- Confidentiality of IoT key: The IoT key is important and utilized to encrypt the messages sent in the IoT network. The confidentiality of IoT key ensures that the key cannot be recovered by the attackers in the key distribution procedure.

- Authentication of IoT key distribution: In the medical IoT network, the key used to encrypt the IoT message will be periodically updated. The key distribution procedure should be authenticated such that the medical nodes can make sure the key update message is indeed sent by its owner (the pre-defined patient).

- Confidentiality of IoT message: The medical nodes in IoT network collects vital physiological data of the patient and the IoT messages sent by the medical nodes are sensitive. Thus, the confidentiality of the IoT message should be guaranteed.

- Authentication of IoT message: The patient should authenticate that the received encrypted IoT message is indeed sent by the claimed medical node in his own medical IoT network.

- Secure against replay attack: The system should guarantee that it can be detected if the previous sent message is replayed by the adversary. 
Table 1: Notations

\begin{tabular}{|c|c|}
\hline Notation & Description \\
\hline$T A$ & trusted authority \\
\hline$P A$ & patient \\
\hline$M N$ & medical Node of IoT \\
\hline$E H R$ & electronic health record \\
\hline$P I D_{P A}$ & the pseudo identity of $P A$ \\
\hline$P I D_{M N}$ & the pseudo identity of $M N$ \\
\hline$\Sigma_{M N}$ & $\left\{P I D_{M N_{1}}, \cdots, P I D_{M N_{n}}\right\}$ \\
\hline$m$ & message sent in IoT \\
\hline$M$ & EHR in big data system \\
\hline$C_{m} / C T$ & ciphertext of $m / M$ \\
\hline$P P / M S K$ & public parameter/master secret key of the system \\
\hline$P K_{P A} / S K_{P A}$ & public/secret key of $P A$ \\
\hline$P K_{M N} / S K_{M N}$ & public/secret key of $M N$ \\
\hline$\kappa$ & security parameter \\
\hline$p$ & a prime number \\
\hline$Z_{p}^{*}$ & $\{1, \cdots, p-1\}$ \\
\hline$i \in[l]$ & $i \in\{1, \cdots, l\}$ \\
\hline$b \in_{R} B$ & $b$ is randomly chosen from $B$ \\
\hline$S E n c / S D e c$ & secure symmetric encryption/decryption \\
\hline $\mathcal{K}$ & symmetric key space of $S E n c / S D e c$ \\
\hline $\operatorname{attr} / S$ & attribute/attribute set \\
\hline$(\mathbb{A}, \rho)$ & access policy \\
\hline$k$ & symmetric key for e-health IoT network \\
\hline$K E A$ & IoT key extraction auxiliary messages \\
\hline$P U Q$ & policy update query \\
\hline$H_{0}$ & secure hash function $H_{0}:\{0,1\}^{*} \rightarrow \mathcal{K}$ \\
\hline$H_{1}$ & secure hash function $H_{i}:\{0,1\}^{*} \rightarrow Z_{p}^{*}$ \\
\hline$K W=\left(w_{1}, \cdots, w_{l_{1}}\right)$ & keyword set extracted from the EHR \\
\hline$Q W=\left(w_{1}, \cdots, w_{l_{2}}\right)$ & keyword set in the policy update query, $l_{2} \leq l_{1}$ \\
\hline
\end{tabular}




\section{The proposed system}

In this section, the privacy-preserving fusion of IoT and big data system is proposed and the notations used are defined in Table 1. The fusion of IoT and big data system consists of 13 algorithms, which are indicated in Figures 2, 3, 4, and 5. Next, we explain the mechanisms for the fusion of medical IoT and big data.

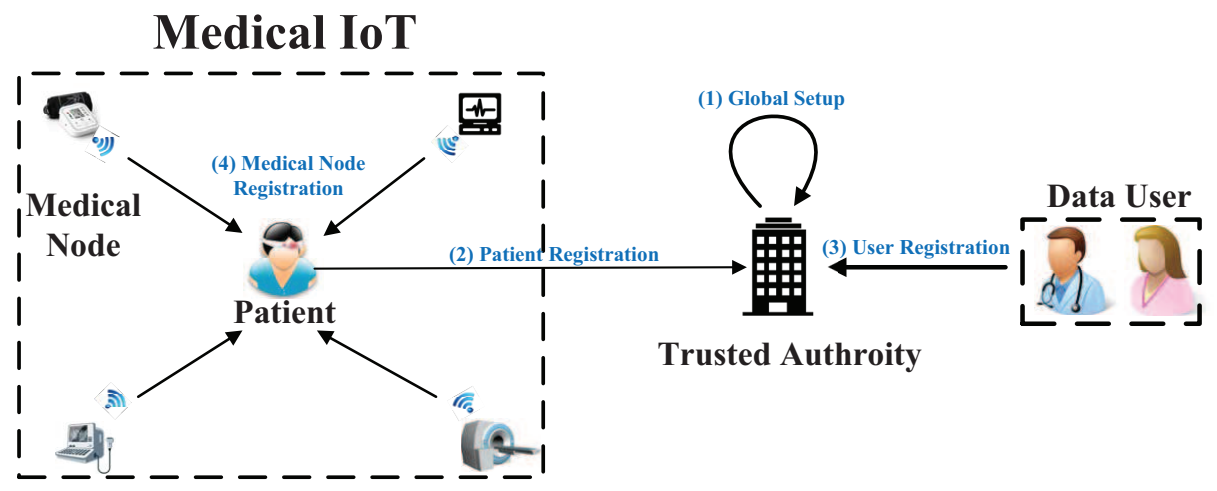

Figure 2: Setup and Registration

As shown in Figure 2 the trusted authority $(T A)$ runs global setup algorithm (Algorithm 1) to generate the public parameter and master secret key for the system. When a patient $(P A)$ registers to the system, $T A$ runs Algorithm 2 to generation the patient's public/secret key pair. When a user $(U)$ registers to the system, $T A$ runs Algorithm 3 to generation the data user's public/secret key pair. When a medical node $(M N)$ in patient $P A$ 's healthcare IoT network registers to the system, $P A$ runs Algorithm 4 to generation the medical node's public/secret key pair.

As shown in Figure 3 the patient $P A$ runs e-health IoT key generation algorithm (Algorithm 5) to realize privacy-preserving IoT key distribution. A key extraction auxiliary message $K E A$ is generated and disseminated to the medical nodes in $P A$ 's IoT network. Then, these nodes runs authenticated e-health IoT key extraction algorithm (Algorithm 6) to recover the IoT key. 


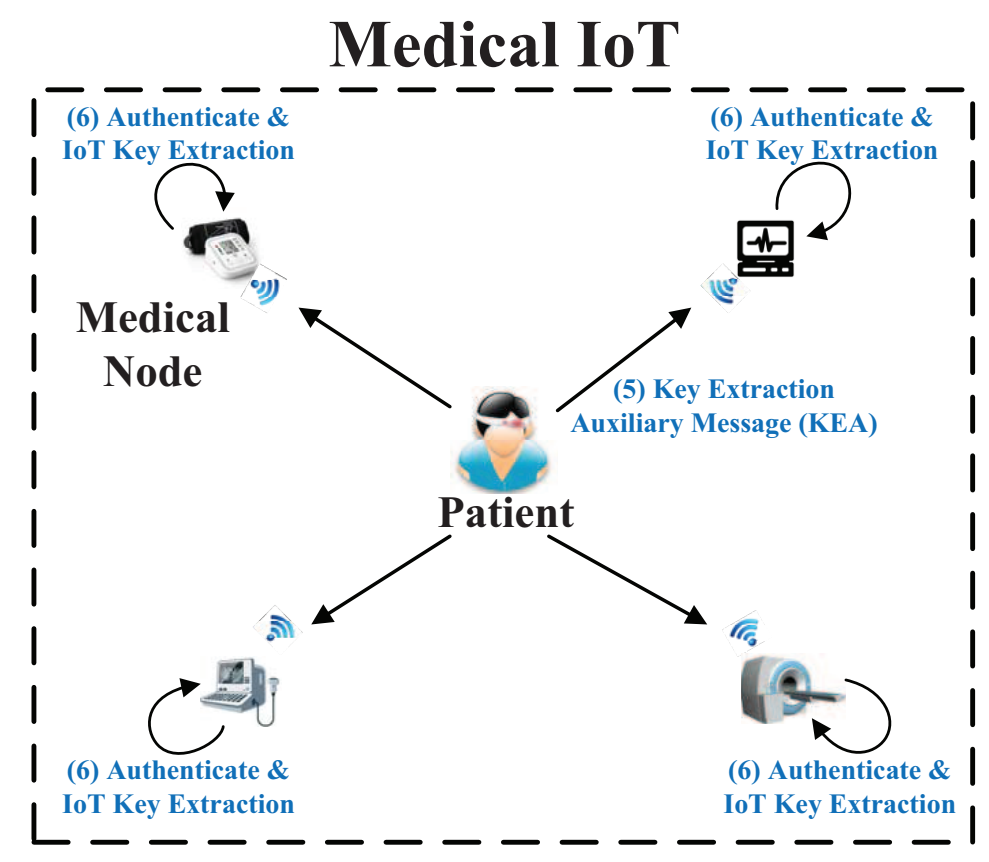

Figure 3: E-health IoT Key Generation and Extraction

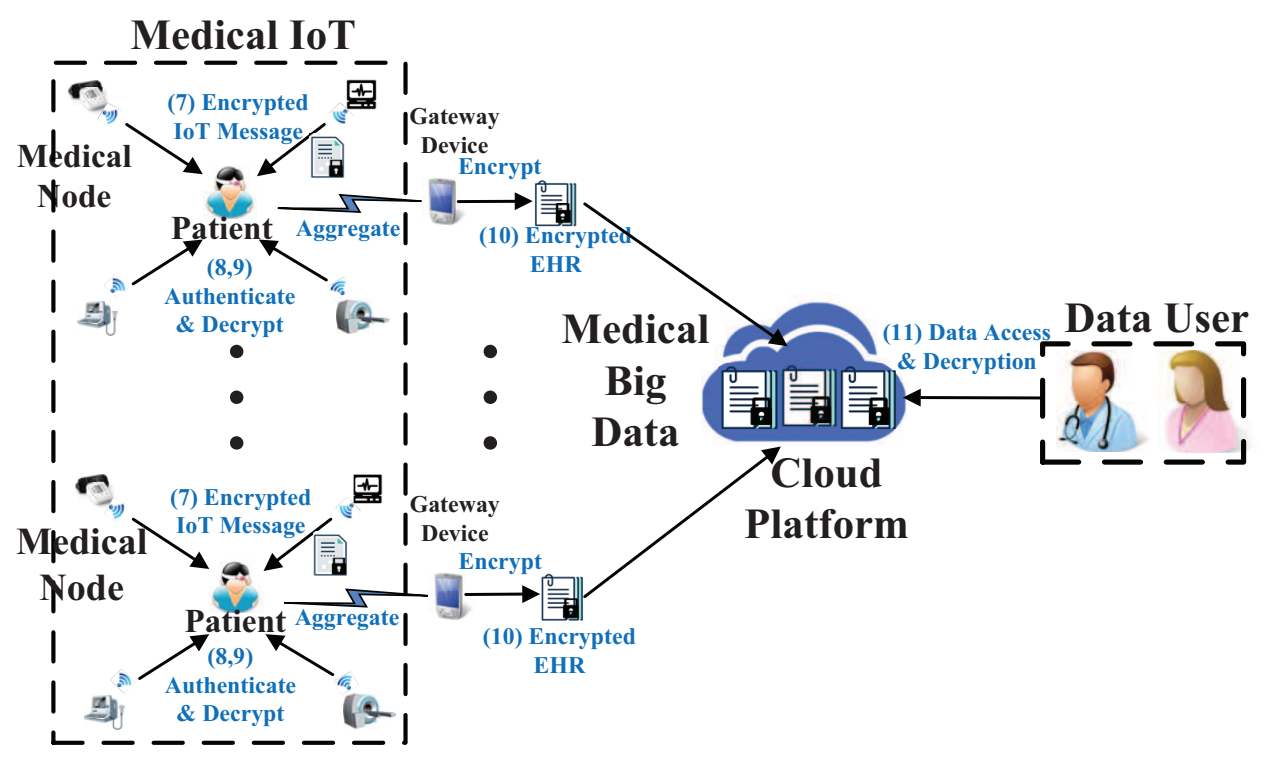

Figure 4: Medical IoT and Big Data Encryption 
As shown in Figure 4, the $M N$ runs IoT message encryption algorithm (Algorithm 7), and utilizes the IoT key to encrypt the IoT message. Receiving the encrypted IoT message, the $P A$ runs e-health IoT message verification and decryption algorithm (Algorithm 8) to verify and recover the IoT message. To accelerate the verification, a batch verification algorithm is designed in Algorithm 9 . Then, the IoT messages are aggregated to EHR file and encrypted by $P A$ using EHR encryption algorithm 10 , where the keywords are extracted and encrypted, and patient defined access policy is exerted to the EHR ciphertext. As the EHRs accumulate, they forms the medical big data system. To save the local storage cost, the medical big data is stored in the cloud platform. The data users with proper attribute secret key is able to access the encrypted EHR data and recover the plaintext using EHR decryption algorithm (Algorithm 11).

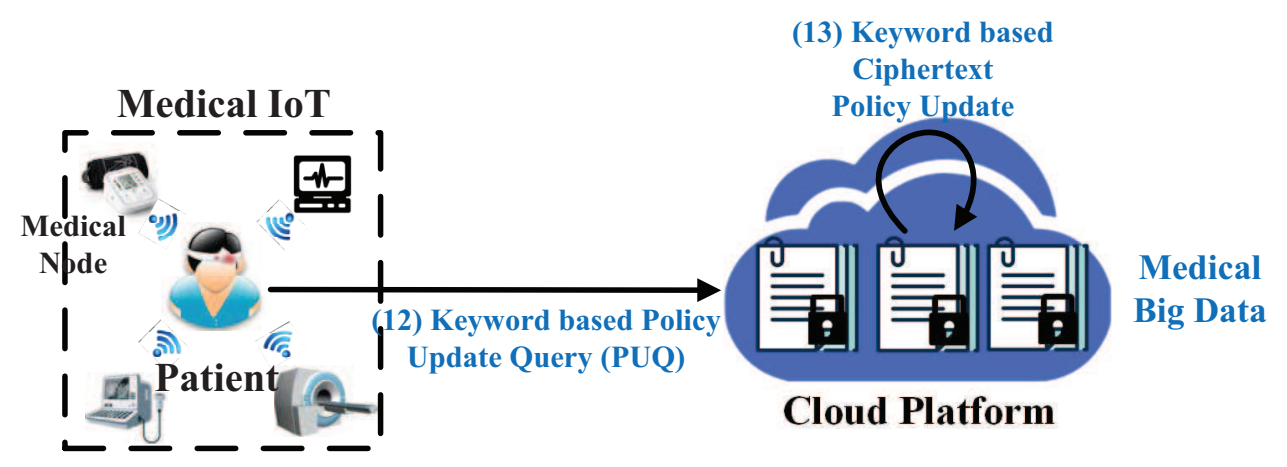

Figure 5: Keyword based Access Policy Update

As shown in Figure 5 if $P A$ wants to update the access policy of the encrypted EHRs, $P A$ runs the keyword match based policy update query algorithm (Algorithm 12) to generate a policy update query PUQ. Receiving the update query, the cloud platform runs the keyword match based ciphertext policy update algorithm (Algorithm 13) to update the ciphertext.

\subsection{Global Setup}

Given the security parameter $\kappa, T A$ runs global setup algorithm (shown in Algorithm 1) to generate the public parameter $P P$ and master secret key $M S K$ 
for the system. $P P$ is public in the system and $M S K$ is confidentially stored by $T A . P P$ is a default input in the following algorithms, which is omitted to simplify the presentation.

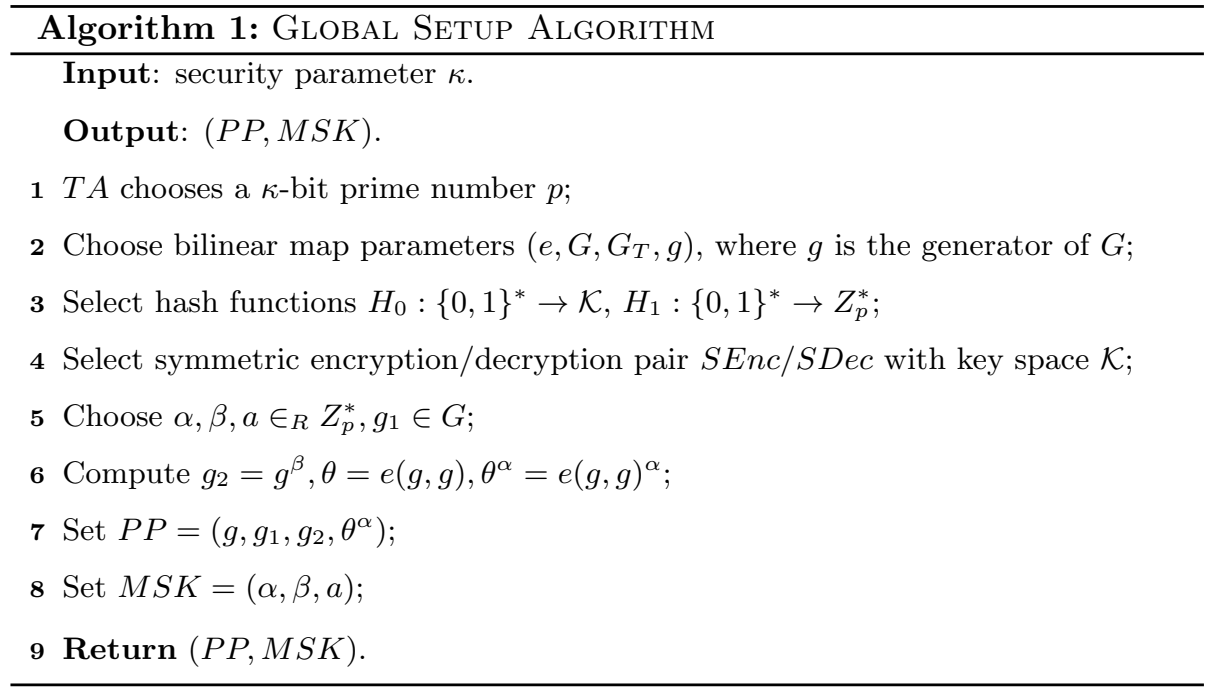

\subsection{Patient Registration}

A patient $P A$ with attribute set $S=\left(a_{t t r}, \cdots, a t t r_{n_{P A}}\right)$ registers to the system. TA runs patient registration algorithm (shown in Algorithm 2 ) to generate $P A$ 's public/secret key pair $P K_{P A} / S K_{P A}$. $T A$ firstly generates $P A$ 's pseudonym identity $P I D_{P A}$ using the master secret key $M S K$ and the symmetric encryption algorithm $S E n c$ (Line 1). $P A$ 's public key $P K_{P A}$ is constructed in Line 2-4 and secret key $S K_{P A}$ is calculated in Line 5-9.

\subsection{User Registration}

A user $U$ with attribute set $S=\left(a_{t t r_{1}}, \cdots, a t t r_{n_{U}}\right)$ registers to the system, who can be the healthcare staffs, patients' friends and relatives. $T A$ runs user registration algorithm (shown in Algorithm 3 to generate $U$ 's public/secret key pair $P K_{U} / S K_{U}$, which is similar to patient registration algorithm (shown in Algorithm 2). 

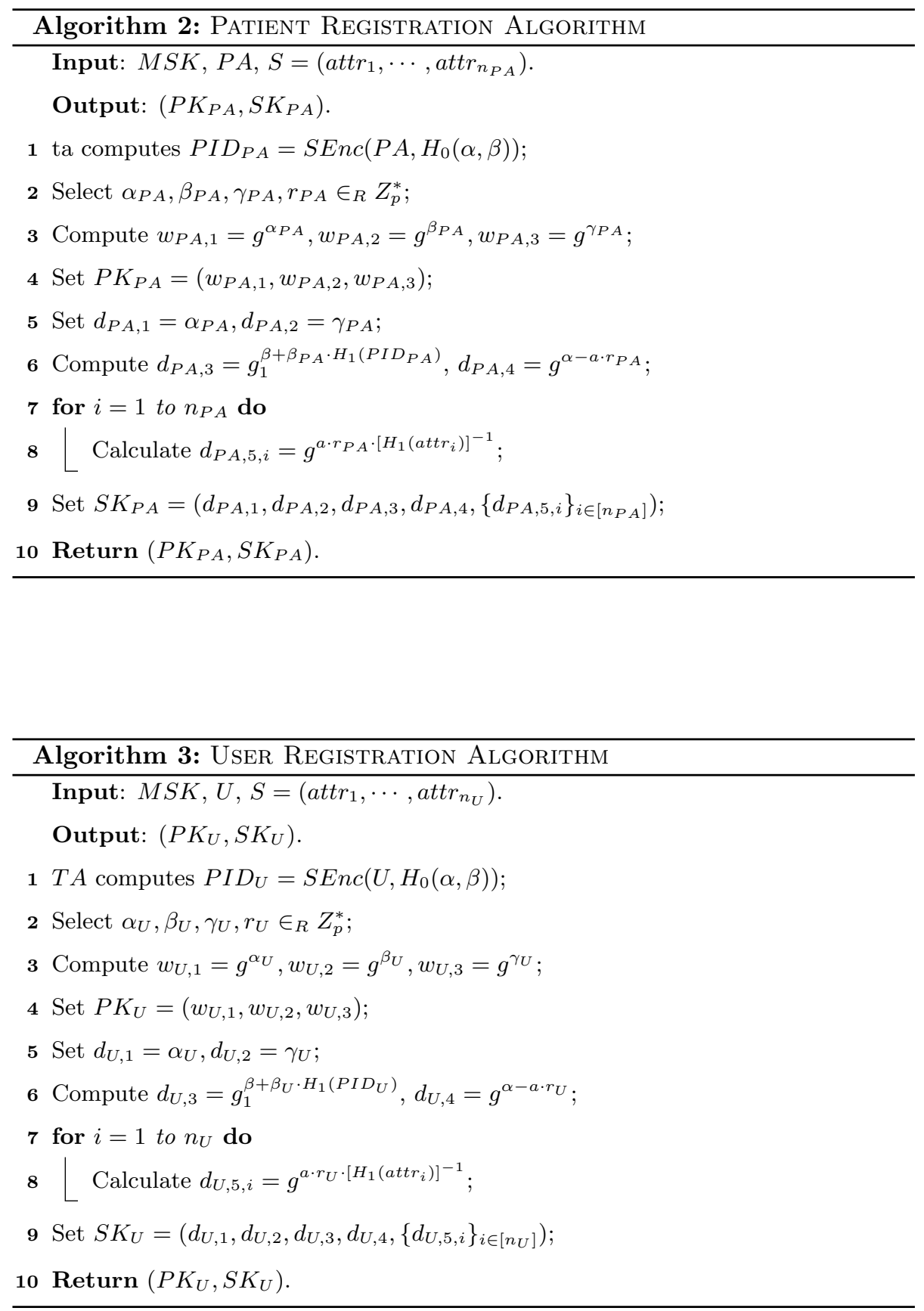


\subsection{Medical Node in IoT Registration}

The medical node $M N$ in patient $P A$ 's healthcare IoT network registers to the system. $P A$ runs medical node in IoT registration algorithm (shown in Algorithm 4 to generate $M N$ 's public/secret key pair $P K_{M N} / S K_{M N}$. $P A$ firstly generates $M N$ 's pseudonym identity $P I D_{M N}$ using $P A$ 's secret key $S K_{P A}$ and the symmetric encryption algorithm SEnc (Line 1). $M N$ 's public key $P K_{M N}$ is constructed in Line 2-3 and secret key $S K_{M N}$ is calculated in Line 4-5.

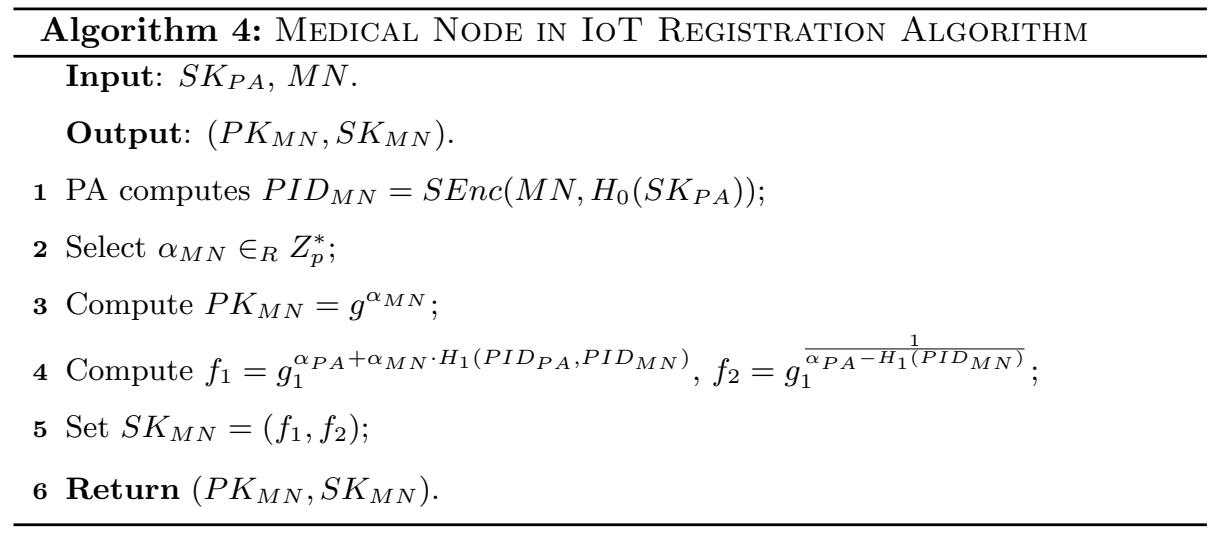

\subsection{E-health IoT Key Generation}

Suppose $\Sigma_{M N}=\left\{P I D_{M N_{1}}, \cdots, P I D_{M N_{n}}\right\}$ is the medical node set of patient $P A$ 's health IoT network. To guarantee the privacy-preserving IoT message delivery, $P A$ runs e-health IoT key generation algorithm (shown in Algorithm 5 to generate an e-health IoT key $k$ (Line 2) and the key extraction auxiliary messages $K E A=\left(b_{1}, b_{2}, b_{3}, b_{4}, T S_{P A}\right)$ using $P A$ 's secret key $S K_{P A}$, where $T S_{P A}$ is the time stamp to prevent the replay attack. Then, $K E A$ is sent to the medical nodes in $\Sigma_{M N}$. It is required that $K E A$ can be authenticated by the $M N_{i}(1 \leq i \leq n)$ in $\Sigma_{M N}$ and the adversary cannot recover $k$ from $K E A$.

\subsection{Authenticated E-health IoT Key Extraction}

When the medical node $M N_{i} \in \Sigma_{M N}(1 \leq i \leq n)$ receives the IoT key extraction auxiliary messages $K E A=\left(b_{1}, b_{2}, b_{3}, b_{4}, T S\right), M N_{i}$ runs authenticated 


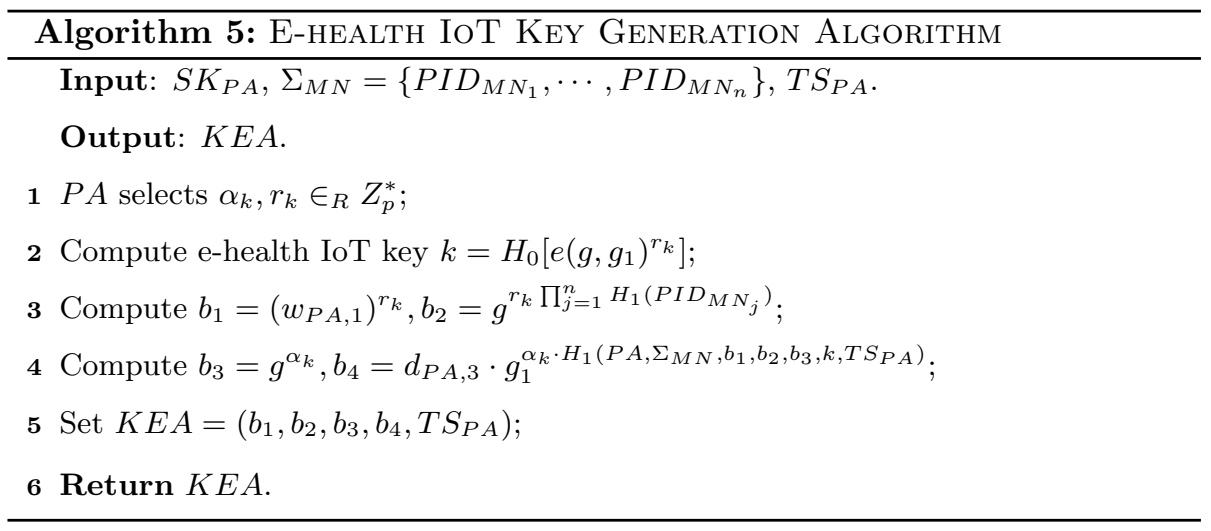

e-health IoT key extraction algorithm (shown in Algorithm 6) to extract the IoT key $k$. In Line 1 , it utilizes its secret key $S K_{M N_{i}}=\left(f_{1}, f_{2}\right)$ to extract the IoT key $k$ by calculating

$$
k=H_{0}\left[e\left(b_{1} \cdot b_{2}^{1 / \prod_{j=1, j \neq i}^{n} H_{1}\left(P I D_{M N_{j}}\right)}, f_{2}\right)\right] .
$$

In Line $2-5, M N_{i}$ checks whether $K E A$ is sent by $P A$, and $k$ is a fresh IoT key generated at time $T S_{P A} . M N_{i}$ verifies whether the following equation holds

$$
e\left(g, b_{4}\right)=e\left(g_{1}, g_{2} \cdot\left(w_{P A, 2}\right)^{H_{1}\left(P I D_{P A}\right)} \cdot b_{3}^{H_{1}\left(P A, \Sigma_{M N}, b_{1}, b_{2}, b_{3}, k, T S_{P A}\right)}\right) .
$$

If it holds, it indicates that $K E A$ and $k$ pass the verification. Otherwise, $K E A$ is rejected and outputs $\perp$.

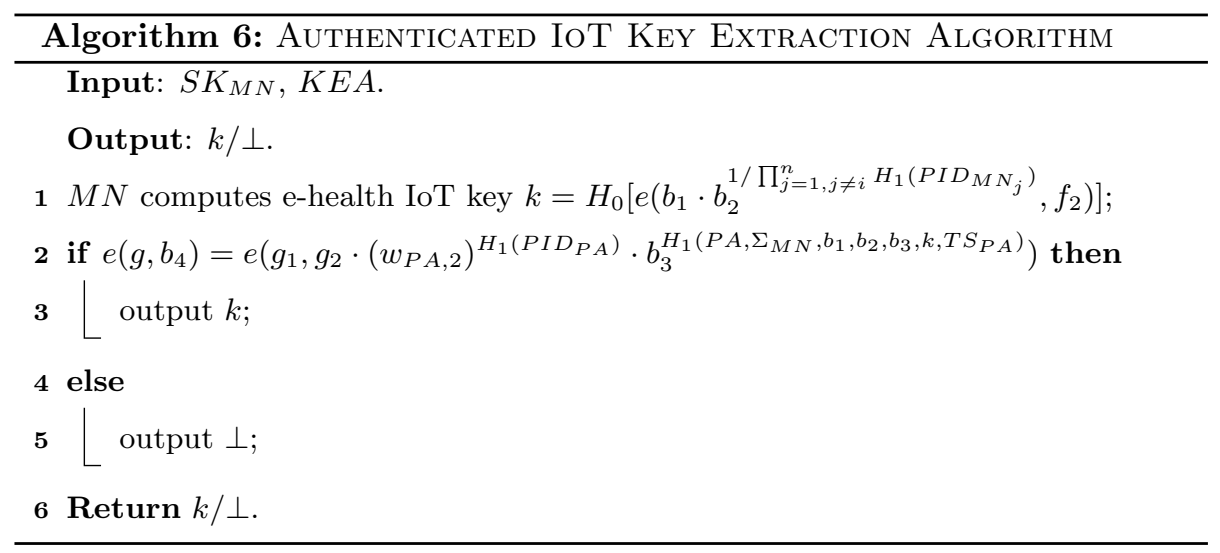




\subsection{E-health IoT Message Encryption}

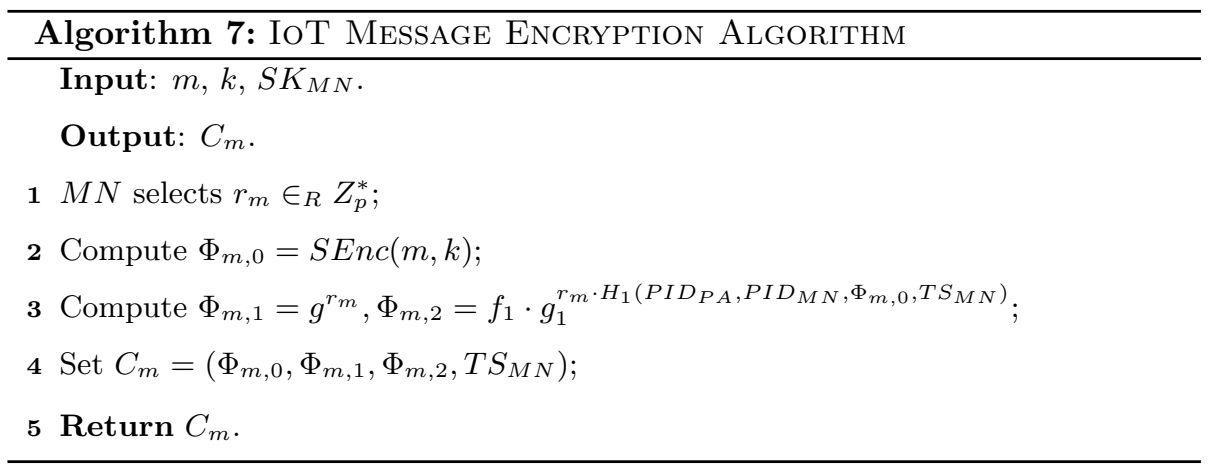

When the IoT medical node $M N$ collects the physiological message $m$ of patient $P A, M N$ runs e-health IoT message encryption algorithm (shown in 77 to encrypt $m$ and generate the ciphertext $C_{m}=\left(\Phi_{m, 0}, \Phi_{m, 1}, \Phi_{m, 2}, T S_{M N}\right)$, where $T S_{M N}$ is the time stamp to generate $C_{m}$ and $C_{m}$ is transmitted to $P A$. It is required that $C_{m}$ can be authenticated by $P A$ and the adversary cannot recover $m$ from $C_{m}$.

\subsection{E-health IoT Message Verification and Decryption}

The messages in the health IoT network are probably transmitted using the wireless channel, which are prone to be captured, tampered or forged by the adversaries. In order to prevent these attackes, it is important for the patient to verify these encrypted IoT messages. When $P A$ receives the IoT ciphertext $C_{m}=\left(\Phi_{m, 0}, \Phi_{m, 1}, \Phi_{m, 2}, T S_{M N}\right)$ from $M N, P A$ runs e-health IoT message verification and decryption algorithm (shown in Algorithm 8 to authenticate $C_{m}$ and decrypt the underlying message $m$. In Line $1, P A$ checks whether $C_{m}$ is a fresh IoT ciphertext sent by $M N$, which is generated at time $T S_{M N}$. $P A$ verifies whether the following equation holds

$$
\begin{aligned}
& e\left(g, \Phi_{m, 2}\right) \\
= & e\left[g_{1}, w_{P A, 1} \cdot\left(P K_{M N}\right)^{H_{1}\left(P I D_{P A}, P I D_{M N}\right)} \cdot\left(\Phi_{m, 1}\right)^{H_{1}\left(P I D_{P A}, P I D_{M N}, \Phi_{m, 0}, T S_{M N}\right)}\right] .
\end{aligned}
$$


If it holds (Line 2), it indicates that $C_{m}$ passes the verification and the algorithm outputs $m=S \operatorname{Dec}\left(\Phi_{m, 0}, k\right)$. Otherwise (Line 3-4), $C_{m}$ is rejected and outputs $\perp$.

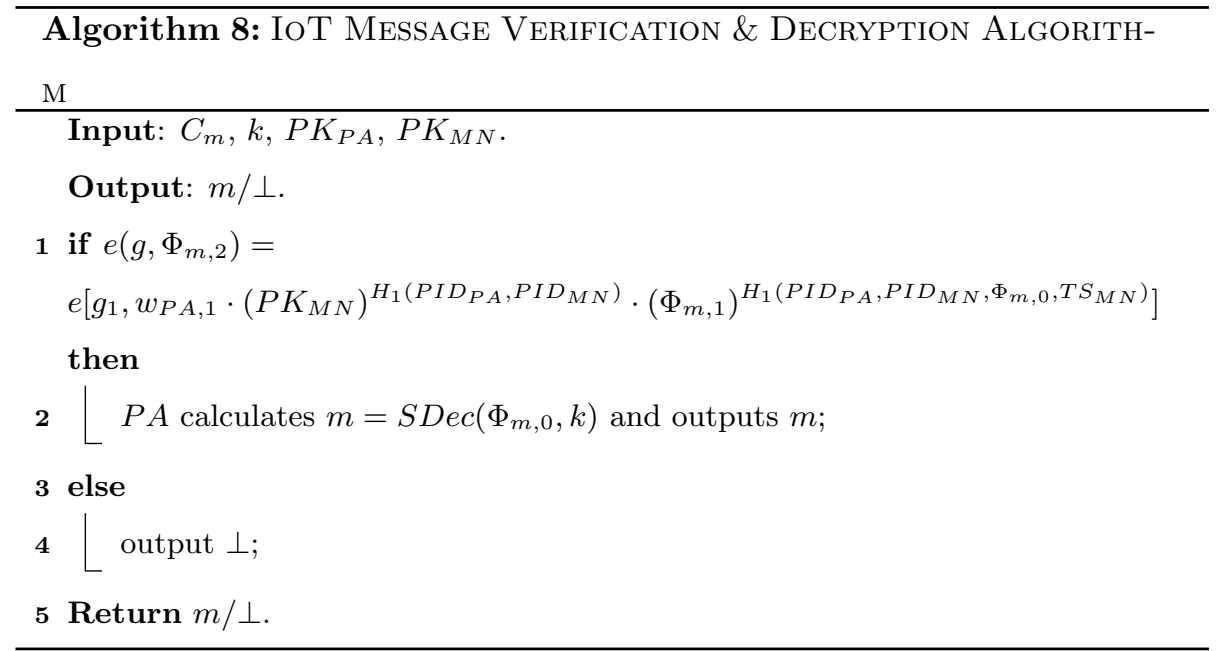

\subsection{E-health IoT Message Batch Verification and Decryption}

When $P A$ receives the IoT ciphertext $C_{m_{i}}=\left(\Phi_{m_{i}, 0}, \Phi_{m_{i}, 1}, \Phi_{m_{i}, 2}, T S_{M N_{i}}\right)$ from $M N_{i}$ for $1 \leq i \leq \tau, P A$ runs e-health IoT message batch verification and decryption algorithm (shown in Algorithm 9 to authenticate $\left(C_{m_{1}}, \cdots, C_{m_{\tau}}\right)$ and decrypt the underlying messages $\left(m_{1}, \cdots, m_{\tau}\right)$.

In Line 1, $P A$ selects random numbers $\delta_{1}, \cdots, \delta_{\tau} \in \in_{R} Z_{p}^{*}$ such that $\Sigma_{i=1}^{\tau} \delta_{i}=1$ mod $p$. In Line 2, $P A$ checks whether $\left(C_{m_{1}}, \cdots, C_{m_{\tau}}\right)$ are fresh IoT ciphertexts sent by $\left(M N_{1}, \cdots, M N_{\tau}\right)$, which are generated at time $\left(T S_{M N_{1}}, \cdots, T S_{M N_{\tau}}\right)$, respectively. $P A$ verifies whether the following equation holds

$$
\begin{gathered}
e\left(g, \prod_{i=1}^{\tau}\left(\Phi_{m_{i}, 2}\right)^{\delta_{i}}\right) \\
=e\left[g_{1}, w_{P A, 1} \prod_{i=1}^{\tau}\left(\left(P K_{M N_{i}}\right)^{\delta_{i} H_{1}\left(P I D_{P A}, P I D_{M N_{i}}\right)} .\right.\right. \\
\left.\left.\left(\Phi_{m_{i}, 1}\right)^{\delta_{i} H_{1}\left(P I D_{P A}, P I D_{M N_{i}}, \Phi_{m_{i}, 0}, T S_{M N_{i}}\right)}\right)\right]
\end{gathered}
$$


If it holds (Line 3-5), it indicates that $\left(C_{m_{1}}, \cdots, C_{m_{\tau}}\right)$ pass the verification and the algorithm outputs $m_{i}=S \operatorname{Dec}\left(\Phi_{m_{i}, 0}, k\right)$ for $1 \leq i \leq \tau$. Otherwise (Line 6-7), the algorithm outputs $\perp$.

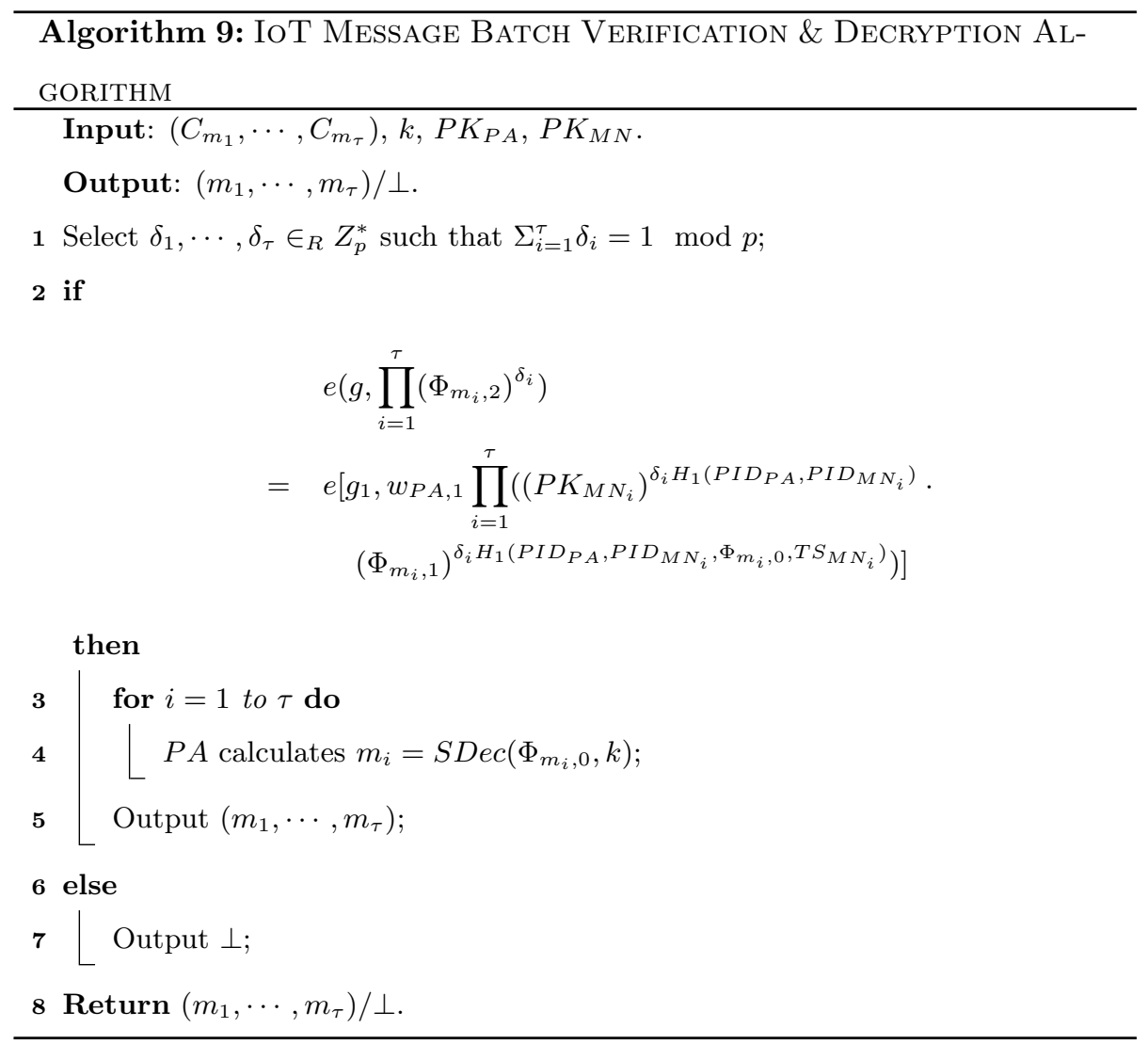

\subsection{Patient Controlled EHR Encryption}

After the IoT messages $\left(m_{1}, \cdots, m_{\tau}\right)$ are received by the patient $P A$, they are aggregated to an EHR file $M . P A$ extracts a keyword set $K W=\left(w_{1}, \cdots, w_{l_{1}}\right)$ to describe $M$ and an access policy $(\mathbb{A}, \rho)$ to designate the permitted data visitors, where $\mathbb{A} \in Z_{p}^{n_{1} \times n_{2}}$ is a matrix and the function $\rho$ maps $\mathbb{A}$ 's rows to attributes.

The EHR encryption algorithm (shown in Algorithm 10 is executed by the patient $P A$ to generate the ciphertext $C T$ of the EHR file $M$ and the keyword 
set $K W=\left(w_{1}, \cdots, w_{l_{1}}\right)$. In Line 1-2, the randomly element $\Upsilon \in_{R} G_{T}$ is selected, $H_{0}(\Upsilon)$ is the symmetric encryption key of the EHR file $M$ and the EHR ciphertext is $C_{M}$. $\Upsilon$ is encrypted in $C_{0}=\Upsilon \cdot \theta^{\alpha s}$. In Line 3-6, the attribute policy $(\mathbb{A}, \rho)$ is encrypted in $\left\{C_{2, i}\right\}_{i \in\left[n_{1}\right]}$ using the LSSS mechanism. In Line 7-9, the keywords in $K W$ are encrypted in $\left\{C_{3, j}\right\}_{j \in\left\{0, \cdots, l_{1}\right\}}$ such that flexible keyword match query is enabled in the next subsection. In Line 10, the ciphertext is set to be $C T=\left((\mathbb{A}, \rho), C_{M}, C_{0}, C_{1},\left\{C_{2, i}\right\}_{i \in\left[n_{1}\right]},\left\{C_{3, j}\right\}_{j \in\left\{0, \cdots, l_{1}\right\}}, C_{4}\right)$.

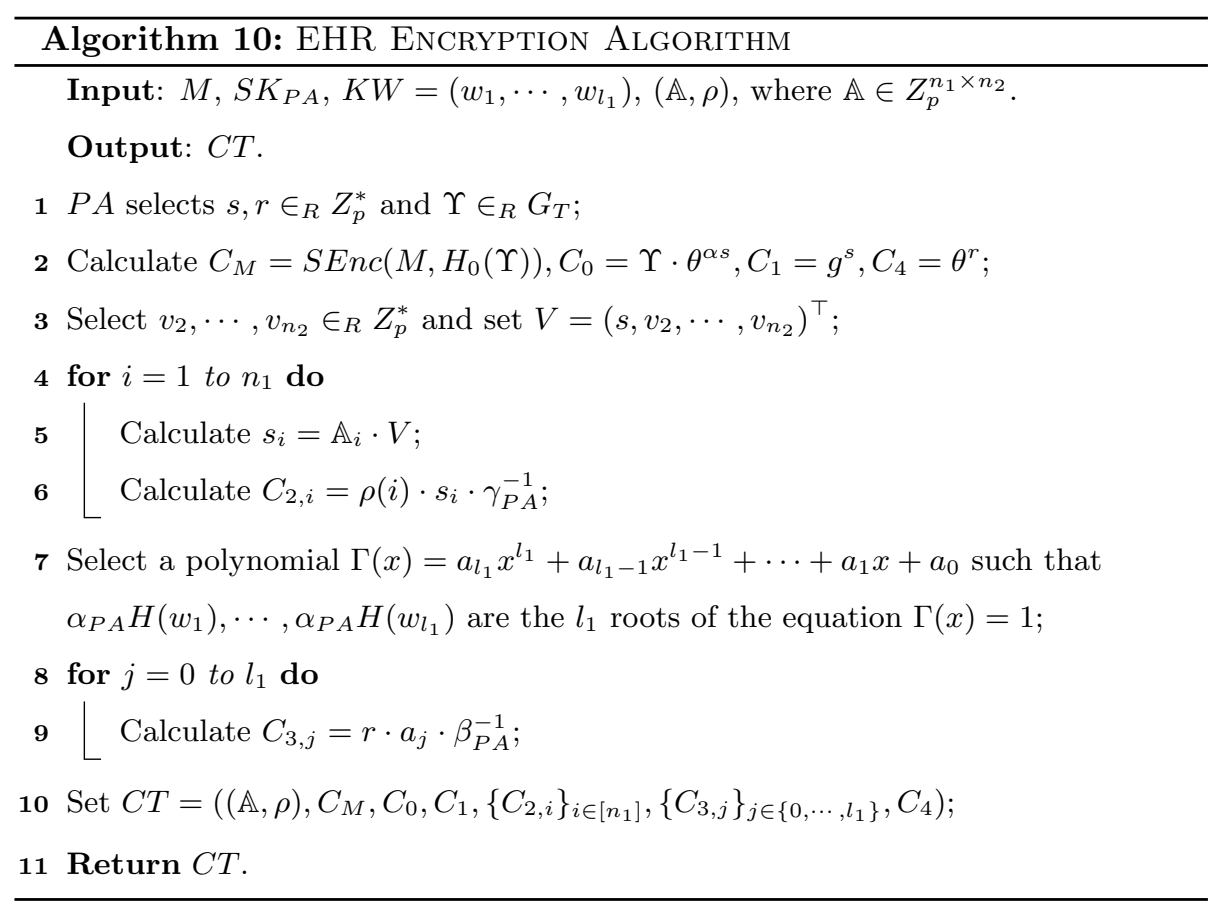

\subsection{EHR Decryption}

When the data user $U$ with attribute set $S$ queries to decrypt patient $P A$ 's EHR ciphertext $C T$ with access policy $(\mathbb{A}, \rho), U$ runs the EHR decryption algorithm (shown in Algorithm 11) to recover the plaintext $M$. If $S$ satisfies $(\mathbb{A}, \rho)$, $U$ utilizes his secret key $S K_{U}$ and LSSS scheme to decrypt $\Upsilon$ and recover $M$. Otherwise, the algorithm outputs $\perp$. 


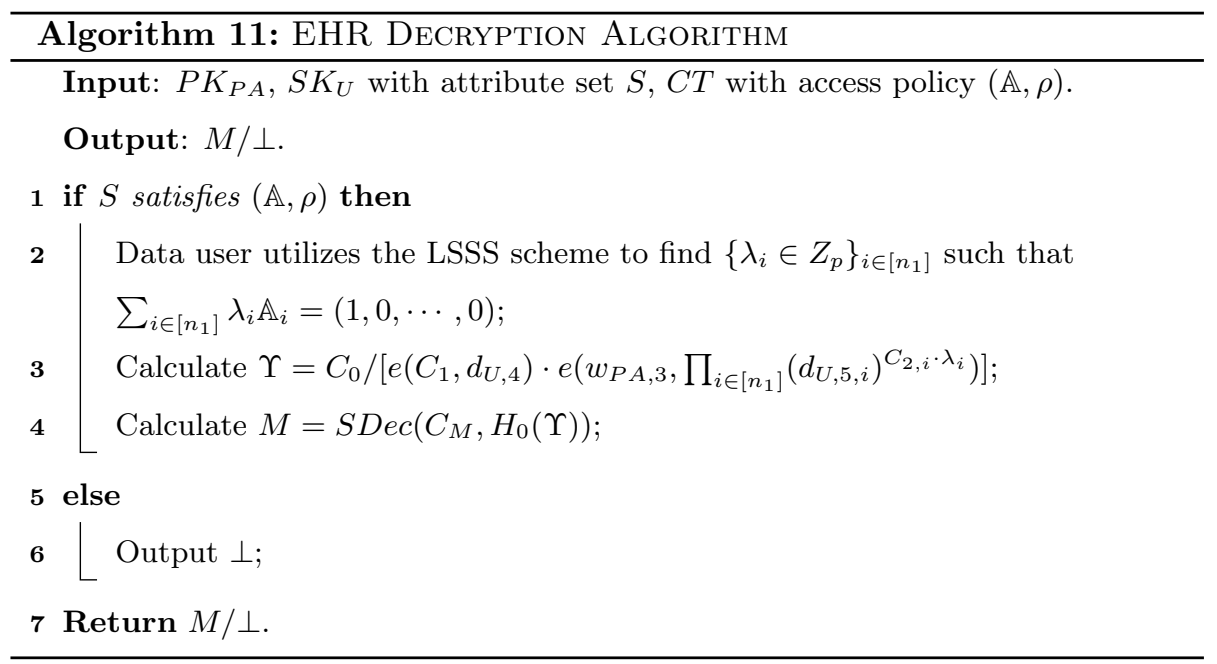

\subsection{Keyword Match based Policy Update Query}

If the patient $P A$ wants to update the access policy of the EHR ciphertext that are stored in the e-health big data system, he runs the keyword match based policy update query algorithm (shown in Algorithm 12 to generate a policy update query $P U Q$, which is submitted to the cloud platform.

Suppose that the original access policy is $(\mathbb{A}, \rho)$ with $\mathbb{A} \in Z_{p}^{n_{1} \times n_{2}}$ and the update access policy is $\left(\mathbb{A}^{\prime}, \rho^{\prime}\right)$ with $\mathbb{A}^{\prime} \in Z_{p}^{n_{1}^{\prime} \times n_{2}^{\prime}}$. In the keyword match based policy update mechanism, the patient $P A$ designates a query keyword set $Q W=$ $\left(w_{\sigma_{1}}, \cdots, w_{\sigma_{l_{2}}}\right)$. Only the ciphertext with keyword set $K W=\left(w_{1}, \cdots, w_{l_{1}}\right)$ that satisfies $Q W \subseteq K W$ will be updated.

In Line $1-4$, the update attribute policy $\left(\mathbb{A}^{\prime}, \rho^{\prime}\right)$ is encrypted in $\left\{C_{2, i}^{\prime}\right\}_{i \in\left[n_{1}^{\prime}\right]}$ using the LSSS mechanism. In Line 5-6, the query keywords in $Q W$ are encrypted in $\left\{q_{j}\right\}_{j \in\left\{0, \cdots, l_{2}\right\}}$. In Line 7 , the policy update query is set to be $P U Q=\left((\mathbb{A}, \rho),\left(\mathbb{A}^{\prime}, \rho^{\prime}\right),\left\{C_{2, i}^{\prime}\right\}_{i \in\left[n_{1}^{\prime}\right]},\left\{q_{j}\right\}_{j \in\left\{0, \cdots, l_{2}\right\}}\right)$.

\subsection{Keyword Match based Ciphertext Policy Update}

Receiving the policy update query $P U Q$ from patient $P A$, the cloud platform runs the keyword match based ciphertext policy update algorithm (shown in Algorithm 13 to update the ciphertext. 


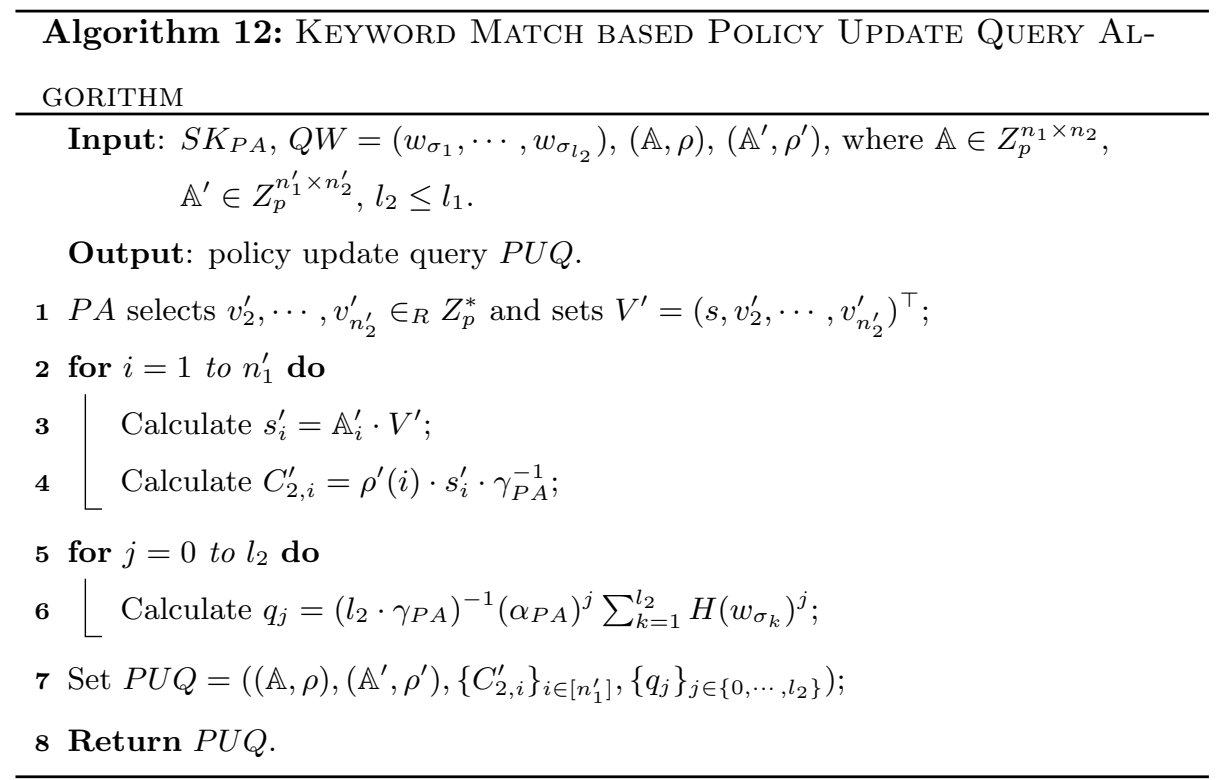

Suppose the ciphertext $C T$ has access policy $(\mathbb{A}, \rho)$ and keyword set $K W=$ $\left(w_{1}, \cdots, w_{l_{1}}\right)$, and $P U Q$ has update access policy $\left(\mathbb{A}^{\prime}, \rho^{\prime}\right)$ and query keyword set $Q W=\left(w_{\sigma_{1}}, \cdots, w_{\sigma_{l_{2}}}\right)$. In Line 1-2, the cloud platform tests whether $Q W \subseteq$ $K W$ by verifying whether the equation $e\left(w_{P A, 3}, w_{P A, 2}\right)^{\sum_{j=0}^{l_{1}}\left(C_{3, j} \cdot q_{j}\right)}=C_{4}$ holds. If it holds, the updated ciphertext is

$$
C T^{\prime}=\left((\mathbb{A}, \rho),\left(\mathbb{A}^{\prime}, \rho^{\prime}\right), C_{M}, C_{0}, C_{1},\left\{C_{2, i}^{\prime}\right\}_{i \in\left[n_{1}^{\prime}\right]},\left\{C_{3, j}\right\}_{j \in\left\{0, \cdots, l_{1}\right\}}, C_{4}\right) .
$$

Otherwise, $C T$ does not satisfy the update requirement. Cloud platform sets $C T^{\prime}=C T$ to indicate that the ciphertext is not updated (Line 3-4).

\section{Correctness Analysis}

The correctness of the algorithms in this system are analyzed below.

\subsection{Correctness of Authenticated IoT Key Extraction Algorithm}

Since $b_{1}=\left(w_{P A, 1}\right)^{r_{k}}, b_{2}=g^{r_{k} \prod_{j=1}^{n} H_{1}\left(P I D_{M N_{j}}\right)}, f_{2}=g_{1}^{\frac{1}{\alpha_{P A}-H_{1}\left(P I D_{M N}\right)}}$, $w_{P A, 1}=g^{\alpha_{P A}}$ and $k=H_{0}\left[e\left(g, g_{1}\right)^{r_{k}}\right]$, we can deduce that

$$
H_{0}\left[e\left(b_{1} \cdot b_{2}^{1 / \prod_{j=1, j \neq i}^{n} H_{1}\left(P I D_{M N_{j}}\right)}, f_{2}\right)\right]
$$




\section{Algorithm 13: Keyword Match Based Ciphertext Policy Update}

Algorithm

Input: $C T$ with access policy $(\mathbb{A}, \rho) \&$ keyword set $K W=\left(w_{1}, \cdots, w_{l_{1}}\right)$,

$P U Q$ with access policy $\left(\mathbb{A}^{\prime}, \rho^{\prime}\right) \&$ query keyword set $Q W=\left(w_{\sigma_{1}}, \cdots, w_{\sigma_{l_{2}}}\right)$.

Output: $C T^{\prime}$.

1 if $e\left(w_{P A, 3}, w_{P A, 2}\right)^{\sum_{j=0}^{l_{1}}\left(C_{3, j} \cdot q_{j}\right)}=C_{4}$ then

2

Cloud sets

$C T^{\prime}=\left((\mathbb{A}, \rho),\left(\mathbb{A}^{\prime}, \rho^{\prime}\right), C_{M}, C_{0}, C_{1},\left\{C_{2, i}^{\prime}\right\}_{i \in\left[n_{1}^{\prime}\right]},\left\{C_{3, j}\right\}_{j \in\left\{0, \cdots, l_{1}\right\}}, C_{4}\right) ;$

3 else

$4 \quad$ Cloud sets $C T^{\prime}=C T$;

5 Return $C T^{\prime}$.

$$
\begin{aligned}
& =H_{0}\left[e\left(\left(w_{P A, 1}\right)^{r_{k}} \cdot\left(g^{r_{k} \prod_{j=1}^{n} H_{1}\left(P I D_{M N_{j}}\right)}\right)^{1 / \prod_{j=1, j \neq i}^{n} H_{1}\left(P I D_{M N_{j}}\right)}, g_{1}^{\frac{1}{\alpha_{P A}-H_{1}\left(P I D_{M N}\right)}}\right)\right] \\
& =H_{0}\left[e\left(g^{r_{k} \cdot \alpha_{P A}} \cdot g^{r_{k} \cdot H_{1}\left(P I D_{M N_{i}}\right)}, g_{1}^{\frac{1}{\alpha_{P A}-H_{1}\left(P I D_{M N}\right)}}\right)\right] \\
& =H_{0}\left[e\left(g, g_{1}\right)^{r_{k}}\right]=k .
\end{aligned}
$$

Since $b_{3}=g^{\alpha_{k}}, b_{4}=d_{P A, 3} \cdot g_{1}^{\alpha_{k} \cdot H_{1}\left(P A, \Sigma_{M N}, b_{1}, b_{2}, b_{3}, k, T S_{P A}\right)}, w_{P A, 2}=g^{\beta_{P A}}$ and $d_{P A, 3}=g_{1}^{\beta+\beta_{P A} \cdot H_{1}\left(P I D_{P A}\right)}$, we can deduce that

$$
\begin{aligned}
& e\left(g, b_{4}\right) \\
= & e\left(g, d_{P A, 3} \cdot g_{1}^{\alpha_{k} \cdot H_{1}\left(P A, \Sigma_{M N}, b_{1}, b_{2}, b_{3}, k, T S_{P A}\right)}\right) \\
= & e\left(g, g_{1}^{\beta+\beta_{P A} \cdot H_{1}\left(P I D_{P A}\right)} \cdot g_{1}^{\alpha_{k} \cdot H_{1}\left(P A, \Sigma_{M N}, b_{1}, b_{2}, b_{3}, k, T S_{P A}\right)}\right) \\
= & e\left(g_{1}, g^{\beta+\beta_{P A} \cdot H_{1}\left(P I D_{P A}\right)} \cdot g^{\alpha_{k} \cdot H_{1}\left(P A, \Sigma_{M N}, b_{1}, b_{2}, b_{3}, k, T S_{P A}\right)}\right) \\
= & e\left(g_{1}, g_{2} \cdot\left(w_{P A, 2}\right)^{H_{1}\left(P I D_{P A}\right)} \cdot b_{3}^{H_{1}\left(P A, \Sigma_{M N}, b_{1}, b_{2}, b_{3}, k, T S_{P A}\right)}\right) .
\end{aligned}
$$

\subsection{Correctness of IoT Message Verification \& Decryption Algorithm}

Since $\Phi_{m, 1}=g^{r_{m}}, \Phi_{m, 2}=f_{1} \cdot g_{1}^{r_{m} \cdot H_{1}\left(P I D_{P A}, P I D_{M N}, \Phi_{m, 0}, T S_{M N}\right)}, w_{P A, 1}=$ $g^{\alpha_{P A}}, f_{1}=g_{1}^{\alpha_{P A}+\alpha_{M N} \cdot H_{1}\left(P I D_{P A}, P I D_{M N}\right)}$ and $P K_{M N}=g^{\alpha_{M N}}$, we can deduce that

$$
e\left(g, \Phi_{m, 2}\right)
$$




$$
\begin{aligned}
& =e\left(g, f_{1} \cdot g_{1}^{r_{m} \cdot H_{1}\left(P I D_{P A}, P I D_{M N}, \Phi_{m, 0}, T S_{M N}\right)}\right) \\
& =e\left(g, g_{1}^{\alpha_{P A}+\alpha_{M N} \cdot H_{1}\left(P I D_{P A}, P I D_{M N}\right)} \cdot g_{1}^{r_{m} \cdot H_{1}\left(P I D_{P A}, P I D_{M N}, \Phi_{m, 0}, T S_{M N}\right)}\right) \\
& =e\left(g_{1}, g^{\alpha_{P A}+\alpha_{M N} \cdot H_{1}\left(P I D_{P A}, P I D_{M N}\right)} \cdot g^{r_{m} \cdot H_{1}\left(P I D_{P A}, P I D_{M N}, \Phi_{m, 0}, T S_{M N}\right)}\right) \\
& =e\left[g_{1}, w_{P A, 1} \cdot\left(P K_{M N}\right)^{H_{1}\left(P I D_{P A}, P I D_{M N}\right)} \cdot\left(\Phi_{m, 1}\right)^{H_{1}\left(P I D_{P A}, P I D_{M N}, \Phi_{m, 0}, T S_{M N}\right)}\right] .
\end{aligned}
$$

\subsection{Correctness of IoT Message Batch Verification and Decryption Algorithm}

Since $\Phi_{m_{i}, 2}=f_{1} \cdot g_{1}^{r_{m_{i}} \cdot H_{1}\left(P I D_{P A}, P I D_{M N_{i}}, \Phi_{m_{i}, 0}, T S_{M N_{i}}\right)}, w_{P A, 1}=g^{\alpha_{P A}}, P K_{M N_{i}}=$ $g^{\alpha_{M N_{i}}}, f_{1}=g_{1}^{\alpha_{P A}+\alpha_{M N_{i}} \cdot H_{1}\left(P I D_{P A}, P I D_{M N_{i}}\right)}$ and $\Phi_{m_{i}, 1}=g^{r_{m_{i}}}$, we can deduce that

$$
\begin{aligned}
& e\left(g, \prod_{i=1}^{\tau}\left(\Phi_{m_{i}, 2}\right)^{\delta_{i}}\right) \\
= & e\left[g, \prod_{i=1}^{\tau}\left(f_{1} \cdot g_{1}^{r_{m_{i}} \cdot H_{1}\left(P I D_{P A}, P I D_{M N_{i}}, \Phi_{m_{i}, 0}, T S_{M N_{i}}\right)}\right)^{\delta_{i}}\right] \\
= & e\left[g, \prod_{i=1}^{\tau}\left(g_{1}^{\alpha_{P A}+\alpha_{M N_{i}} \cdot H_{1}\left(P I D_{P A}, P I D_{M N_{i}}\right)}\right.\right. \\
& \left.\left.\cdot g_{1}^{r_{m} \cdot H_{1}\left(P I D_{P A}, P I D_{M N_{i}}, \Phi_{m_{i}, 0}, T S_{M N_{i}}\right)}\right)^{\delta_{i}}\right] \\
= & e\left[g_{1}, \prod_{i=1}^{\tau}\left(g^{\alpha_{P A}+\alpha_{M N_{i}} \cdot H_{1}\left(P I D_{P A}, P I D_{M N_{i}}\right)}\right.\right. \\
& \cdot g^{\left.\left.r_{m_{i}} \cdot H_{1}\left(P I D_{P A}, P I D_{M N_{i}}, \Phi_{m_{i}, 0}, T S_{M N_{i}}\right)\right)^{\delta_{i}}\right]} \\
= & e\left[g_{1}, \cdot \prod_{i=1}^{\tau}\left(w_{1}^{\delta_{i}} \cdot\left(P K_{M N_{i}}\right)^{\delta_{i} \cdot H_{1}\left(P I D_{P A}, P I D_{M N_{i}}\right)}\right.\right. \\
& \left.\left.\cdot\left(\Phi_{m_{i}, 1}\right)^{\delta_{i} \cdot H_{1}\left(P I D_{P A}, P I D_{M N_{i}}, \Phi_{m_{i}, 0}, T S_{M N_{i}}\right)}\right)\right] \\
= & e\left[g_{1}, w_{P A, 1} \cdot \prod_{i=1}^{\tau}\left(\left(P K_{M N_{i}}\right)^{\delta_{i} \cdot H_{1}\left(P I D_{P A}, P I D_{M N_{i}}\right)}\right.\right. \\
& \left.\left.\cdot\left(\Phi_{m_{i}, 1}\right)^{\delta_{i} \cdot H_{1}\left(P I D_{P A}, P I D_{M N_{i}}, \Phi_{m_{i}, 0}, T S_{M N_{i}}\right)}\right)\right] .
\end{aligned}
$$

5.4. Correctness of Keyword Match Based Ciphertext Policy Update Algorithm

Since $C_{3, j}=r \cdot a_{j} \cdot \beta_{P A}^{-1}, q_{j}=\left(l_{2} \cdot \gamma_{P A}\right)^{-1}\left(\alpha_{P A}\right)^{j} \sum_{k=1}^{l_{2}} H\left(w_{k}\right)^{j}, w_{P A, 2}=g^{\beta_{P A}}$ and $w_{P A, 3}=g^{\gamma_{P A}}$, we can deduce that

$$
\begin{aligned}
& e\left(w_{P A, 3}, w_{P A, 2}\right)^{\sum_{j=0}^{l_{1}}\left(C_{3, j} \cdot q_{j}\right)} \\
= & e\left(g^{\gamma_{P A}}, g^{\beta_{P A}}\right)^{\sum_{j=0}^{l_{1}}\left(r \cdot a_{j} \cdot \beta_{P A}^{-1}\right) \cdot\left(l_{2} \cdot \gamma_{P A}\right)^{-1}\left(\alpha_{P A}\right)^{j} \sum_{k=1}^{l_{2}} H\left(w_{k}\right)^{j}}
\end{aligned}
$$




$$
\begin{aligned}
& =e(g, g)^{r \cdot l_{2}^{-1} \cdot \sum_{j=0}^{l_{1}} \cdot a_{j} \cdot\left(\alpha_{P A}\right)^{j} \sum_{k=1}^{l_{2}} H\left(w_{k}\right)^{j}} \\
& =e(g, g)^{r \cdot l_{2}^{-1} \cdot \sum_{k=1}^{l_{2}}\left(\sum_{j=0}^{l_{1}} \cdot a_{j} \cdot\left(\alpha_{P A} H\left(w_{k}\right)\right)^{j}\right)} \\
& =e(g, g)^{r \cdot l_{2}^{-1} \cdot \sum_{k=1}^{l_{2}} \Gamma\left(\alpha_{P A} H\left(w_{k}\right)\right)} \\
& =e(g, g)^{r \cdot l_{2}^{-1} \cdot \sum_{k=1}^{l_{2}} 1}=e(g, g)^{r}=C_{4} .
\end{aligned}
$$

\subsection{Correctness of EHR Decryption Algorithm}

Since $C_{1}=g^{s}, C_{2, i}=\rho(i) \cdot s_{i} \cdot \gamma_{P A}^{-1}, w_{P A, 3}=g^{\gamma_{P A}}, d_{U, 4}=g^{\alpha-a \cdot r_{U}}$ and $d_{U, 5, i}=g^{a \cdot r_{U} \cdot H_{1}\left(a t t r_{i}\right)}$, we can deduce that

$$
\begin{aligned}
& e\left(C_{1}, d_{U, 4}\right) \cdot e\left(w_{P A, 3}, \prod_{i \in\left[n_{1}\right]}\left(d_{U, 5, i}\right)^{C_{2, i} \cdot \lambda_{i}}\right) \\
= & e\left(g^{s}, g^{\alpha-a \cdot r_{U}}\right) \cdot e\left(g^{\gamma_{P A}}, \prod_{i \in\left[n_{1}\right]}\left(g^{a \cdot r_{U} \cdot\left[H_{1}\left(a t t r_{i}\right)\right]^{-1}}\right)^{\rho(i) \cdot s_{i} \cdot \gamma_{P A}^{-1} \cdot \lambda_{i}}\right) \\
= & e\left(g^{s}, g^{\alpha-a \cdot r_{U}}\right) \cdot e\left(g^{\gamma_{P A}}, g^{a \cdot r_{U} \cdot \gamma_{P A}^{-1} \cdot \sum_{i \in\left[n_{1}\right]}\left(\left[H_{1}\left(a t t r_{i}\right)\right]^{-1} \cdot \rho(i) \cdot s_{i} \cdot \lambda_{i}\right)}\right) \\
= & e\left(g^{s}, g^{\alpha-a \cdot r_{U}}\right) \cdot e\left(g, g^{a \cdot r_{U} \cdot s}\right)=\theta^{\alpha s} .
\end{aligned}
$$

\section{Security Proof}

Theorem 6.1. This system for the fusion of IoT and big data is indistinguishable against chosen plaintext and chosen keyword attack (IND-CPCKA) assuming that the decisional bilinear Diffie-Hellman (DBDH) assumption is intractable.

Proof. Suppose that the adversary $\mathcal{A}$ is given a tuple $\left(g, g^{a}, g^{b}, g^{s}, Z\right)$ as an instance of the DBDH problem, where $Z$ is either $e(g, g)^{a b s}$ or a random number in $G_{T}$. The interactive game between $\mathcal{A}$ and $\mathcal{C}$ proceeds as below.

- Setup. $\mathcal{C}$ chooses $\alpha^{\prime}, \beta \in_{R} Z_{p}^{*}, g_{1} \in_{R} G$ and implicitly sets $\alpha=\alpha^{\prime}+a b$. Compute $g_{2}=g^{\beta}, \theta^{\alpha}=e\left(g^{a}, g^{b}\right) \cdot e(g, g)^{\alpha^{\prime}}=e(g, g)^{\alpha}$. $\mathcal{C}$ sends the public parameter $P P=\left(g, g_{1}, g_{2}, \theta^{\alpha}\right)$ to $\mathcal{A}$.

- Phase 1. The following queries are issued by the adversary $\mathcal{A}$. 
- Patient's secret key query: $\mathcal{A}$ queries on the patient PA's attribute secret key with attribute set $S=\left(a t t r_{1} \cdots a t t r_{n_{P A}}\right) . \mathcal{C}$ computes $P I D_{P A}=\operatorname{SEnc}\left(P A, H_{0}(\alpha, \beta)\right)$, selects $\alpha_{P A}, \beta_{P A}, \gamma_{P A}, r_{P A}^{\prime} \in_{R} Z_{p}^{*}$ and computes $d_{P A, 1}=\alpha_{P A}, d_{P A, 2}=\gamma_{P A}, d_{P A, 3}=g_{1}^{\beta+\beta_{P A} \cdot H_{1}\left(P I D_{P A}\right)}$, $d_{P A, 5, i}=\left(g^{a}\right)^{r_{P A} \cdot H_{1}\left(a t t r_{i}\right)}$ for $1 \leq i \leq n_{P A}$. Implicitly set $r_{P A}=$ $b+r_{P A}^{\prime}$ and calculate

$d_{P A, 4}=g^{\alpha-a \cdot r_{P A}}=g^{\left(\alpha^{\prime}+a b\right)-a \cdot\left(b+r_{P A}^{\prime}\right)}=g^{\alpha^{\prime}-a r_{P A}^{\prime}}=g^{\alpha^{\prime}} \cdot\left(g^{a}\right)^{-r_{P A}^{\prime}}$.

$P A$ 's secret key $S K_{P A}=\left(d_{P A, 1}, d_{P A, 2}, d_{P A, 3}, d_{P A, 4},\left\{d_{P A, 5, i}\right\}_{i \in\left[n_{P A}\right]}\right)$ is sent to $\mathcal{A}$.

- Users's secret key query: $\mathcal{A}$ queries on the user U's attribute secret key with attribute set $S=\left(a t t r_{1} \cdots a t t r_{n_{U}}\right)$. $\mathcal{C}$ computes $P I D_{U}=$ $\operatorname{SEnc}\left(U, H_{0}(\alpha, \beta)\right)$, selects $\alpha_{U}, \beta_{U}, \gamma_{U}, r_{U}^{\prime} \in_{R} Z_{p}^{*}$ and computes $d_{U, 1}=$ $\alpha_{U}, d_{U, 2}=\gamma_{U}, d_{U, 3}=g_{1}^{\beta+\beta_{U} \cdot H_{1}\left(P I D_{U}\right)}, d_{U, 5, i}=\left(g^{a}\right)^{r_{U} \cdot H_{1}\left(a t t r_{i}\right)}$ for $1 \leq i \leq n_{U}$. Implicitly set $r_{U}=b+r_{U}^{\prime}$ and calculate

$$
d_{U, 4}=g^{\alpha-a \cdot r_{U}}=g^{\left(\alpha^{\prime}+a b\right)-a \cdot\left(b+r_{U}^{\prime}\right)}=g^{\alpha^{\prime}-a r_{U}^{\prime}}=g^{\alpha^{\prime}} \cdot\left(g^{a}\right)^{-r_{U}^{\prime}} .
$$

U's secret key $S K_{U}=\left(d_{U, 1}, d_{U, 2}, d_{U, 3}, d_{U, 4},\left\{d_{U, 5, i}\right\}_{i \in\left[n_{U}\right]}\right)$ is sent to $\mathcal{A}$.

- Mobile node's secret key query: $\mathcal{A}$ queries on the mobile node $M N$ 's secret key, which belongs to the patient PA's IoT network. Assume that $\mathcal{A}$ has queried $P A$ 's secret key $S K_{P A}$. Then, $\mathcal{C}$ computes $P I D_{M N}=S E n c\left(M N, H_{0}\left(S K_{P A}\right)\right)$, selects $\alpha_{M N} \in_{R} Z_{p}^{*}$ and computes $f_{1}=g_{1}^{\alpha_{P A}+\alpha_{M N} \cdot H_{1}\left(P I D_{P A}, P I D_{M N}\right)}, f_{2}=g_{1}^{\frac{1}{\alpha_{P A}-H_{1}\left(P I D_{M N}\right)}}$ $M N$ 's secret key $S K_{M N}=\left(f_{1}, f_{2}\right)$ is sent to $\mathcal{A}$.

- Keyword match based policy update query: $\mathcal{A}$ queries on the patient $P A$, keyword set $Q W=\left(w_{\sigma_{1}}, \cdots, w_{\sigma_{l_{2}}}\right)$ and access policies $(\mathbb{A}, \rho),\left(\mathbb{A}^{\prime}, \rho^{\prime}\right), \mathcal{C}$ firstly constructs $P A$ 's secret key $S K_{P A}$ as in the "Patient's secret key query". Then, $\mathcal{C}$ selects $s_{i}^{\prime} \in_{R} Z_{p}^{*}$ for $1 \leq i \leq n_{1}$, and calculates $C_{2, i}^{\prime}=\rho^{\prime}(i) \cdot s_{i}^{\prime} \cdot \gamma_{P A}^{-1}$ for $1 \leq i \leq n_{1}$ and 
$q_{j}=\left(l_{2} \cdot \gamma_{P A}\right)^{-1}\left(\alpha_{P A}\right)^{j} \sum_{k=1}^{l_{2}} H\left(w_{\sigma_{k}}\right)^{j}$ for $0 \leq j \leq n_{2}$. Then, the policy update query $P U Q=\left((\mathbb{A}, \rho),\left(\mathbb{A}^{\prime}, \rho^{\prime}\right),\left\{C_{2, i}^{\prime}\right\}_{i \in\left[n_{1}^{\prime}\right]},\left\{q_{j}\right\}_{j \in\left\{0, \cdots, l_{2}\right\}}\right)$ is returned to $\mathcal{A}$.

- Challenge: $\mathcal{A}$ sends to $\mathcal{C}$ a challenge access policy $\left(\mathbb{A}^{*}, \rho^{*}\right)$, a challenge patient identity $P A^{*}$, a challenge EHR $M^{*}$, two EHR encryption keys $\left(\Upsilon_{0}^{*}, \Upsilon_{1}^{*}\right)$, and two challenge keyword sets $\left(K W_{0}^{*}, K W_{1}^{*}\right)$, where $\mathbb{A}^{*} \in$ $Z_{p}^{n_{1}^{*} \times n_{2}^{*}}$ and $\rho^{*}$ maps $\mathbb{A}^{*}$ s s rows to attributes. It is required that the secret key of the attribute set $S$ that satisfies the challenge access policy $\left(\mathbb{A}^{*}, \rho^{*}\right)$ is not queried in phase 1 . Moreover, the secret key of $P A^{*}$ is not queried. The challenge EHR ciphertext is constructed below.

$\mathcal{C}$ picks $r^{*}, \gamma_{P A^{*}} \in_{R} Z_{p}^{*}$ and $s_{i}^{*} \in_{R} Z_{p}^{*}$ for $1 \leq i \leq n_{1}^{*}$. $\mathcal{C}$ flips random coins $\mu_{1}, \mu_{2} \in\{0,1\}$ and constructs

$$
\begin{aligned}
C_{M}^{*} & =\operatorname{SEnc}\left(M^{*}, H_{0}\left(\Upsilon_{\mu_{1}}^{*}\right)\right), C_{0}^{*}=\Upsilon_{\mu_{1}}^{*} \cdot Z \cdot e\left(g^{\alpha^{\prime}}, g^{s}\right), \\
C_{1}^{*} & =g^{s}, \quad C_{4}^{*}=\theta^{r} \\
C_{2, i}^{*} & =\rho(i)^{*} \cdot s_{i}^{*} \cdot\left(\gamma_{P A}^{*}\right)^{-1}, 1 \leq i \leq n_{1}^{*},
\end{aligned}
$$

Suppose the challenge keyword set $K W_{\mu_{2}}^{*}=\left(w_{1}^{*}, \cdots, w_{l_{1}}^{*}\right)$. $\mathcal{C}$ selects a polynomial $\Gamma^{*}(x)=a_{l_{1}}^{*} x^{l_{1}}+a_{l_{1}-1}^{*} x^{l_{1}-1}+\cdots+a_{1}^{*} x+a_{0}^{*}$ such that $\alpha_{P A^{*}} H\left(w_{1}^{*}\right), \cdots, \alpha_{P A^{*}} H\left(w_{l_{1}}^{*}\right)$ are the $l_{1}$ roots of the equation $\Gamma^{*}(x)=1$. $\mathcal{C}$ calculates $C_{3, j^{*}}=r^{*} \cdot a_{j}^{*} \cdot \beta_{P A^{*}}$ for $0 \leq i \leq l_{1}$. The challenge EHR ciphertext $C T^{*}=\left(\left(\mathbb{A}^{*}, \rho^{*}\right), C_{M}^{*}, C_{0}^{*}, C_{1}^{*},\left\{C_{2, i}^{*}\right\}_{i \in\left[n_{1}\right]},\left\{C_{3, j}^{*}\right\}_{j \in\left\{0, \cdots, l_{1}\right\}}, C_{4}^{*}\right)$ is sent to $\mathcal{A}$.

It is obvious that if $Z=e(g, g)^{a b s}$, then

$$
\begin{aligned}
C_{0}^{*} & =\Upsilon_{\mu_{1}}^{*} \cdot Z \cdot e\left(g^{\alpha^{\prime}}, g^{s}\right) \\
& =\Upsilon_{\mu_{1}}^{*} \cdot e(g, g)^{a b s} \cdot e\left(g^{\alpha^{\prime}}, g^{s}\right) \\
& =\Upsilon_{\mu_{1}}^{*} \cdot e\left(g^{a b+\alpha^{\prime}}, g^{s}\right) \\
& =\Upsilon_{\mu_{1}}^{*} \cdot e(g, g)^{\alpha s} .
\end{aligned}
$$

- Phase 2: It is the same as in Phase 1 except that the secret key of the attribute set $S$ that satisfies the challenge access policy $\left(\mathbb{A}^{*}, \rho^{*}\right)$ is not 
allowed to be queried. In addition, the secret key of $P A^{*}$ is not allowed to be queried.

- Guess: Finally, $\mathcal{A}$ outputs $\mu_{1}^{\prime}, \mu_{2}^{\prime} \in\{0,1\}$. If $\mu_{1}^{\prime}=\mu_{1}$ and $\mu_{2}^{\prime}=\mu_{2}, \mathcal{A}$ wins the game. Then, $\mathcal{C}$ could solve the $\mathrm{DBDH}$ problem by distinguishing $Z=e(g, g)^{a b s}$ or $Z$ is a random element in $G_{T}$.

\section{Security Requirements Analysis}

This IoT and big data fusion system achieves the security requirements defined in Section 3.3

\subsection{Patient anonymity and traceability}

When a patient $P A$ registers to the system in Subsection 4.2 , an anonymous identity $P I D_{P A}=\operatorname{SEnc}\left(P A, H_{0}(\alpha, \beta)\right)$ is generated by ta, where $\alpha, \beta$ are the elements in the master secret key $M S K$. Since $M S K$ is kept secret by ta, no adversary can recover patient's real identity $P A$ from the anonymous identity $P I D_{P A}$ due to the security of the symmetric encryption $S E n c$ algorithm. If a patient is found misbehaving, $T A$ can trace his real identity by calculating $P A=S \operatorname{Dec}\left(P I D_{P A}, H_{0}(\alpha, \beta)\right)$. Thus, the patient anonymity and traceability are guaranteed.

\subsection{User anonymity and traceability}

The analysis of user anonymity and traceability is similar to that in Subsection 7.1, which is omitted here.

\subsection{Medical node anonymity and traceability}

When a medical node $M N$ in patient $P A$ 's health IoT network registers to the system in Subsection 4.4 an anonymous identity $P I D_{M N}$ is generated by $P A$ through calculating $P I D_{M N}=S E n c\left(M N, H_{0}\left(S K_{P A}\right)\right)$, where $S K_{P A}$ is $P A$ 's secret key. Since $S K_{P A}$ is kept secret by $P A$, no adversary can recover medical nodes's real identity $M N$ from the anonymous identity $P I D_{M N}$ 
due to the security of the symmetric encryption $S E n c$ algorithm. If a medical node is found misbehaving, $P A$ can trace its real identity by calculating $M N=S \operatorname{Dec}\left(P I D_{M N}, H_{0}\left(S K_{P A}\right)\right)$. Thus, the medical node anonymity and traceability are guaranteed.

\subsection{Confidentiality of IoT key}

In the e-health IoT key generation algorithm (shown in Subsection 4.5), the IoT key is constructed as $k=H_{0}\left[e\left(g, g_{1}\right)^{r_{k}}\right]$, where $r_{k} \in_{R} Z_{p}^{*}$. The key extraction auxiliary message $K E A$ is constructed as $K E A=\left(b_{1}, b_{2}, b_{3}, b_{4}, T S_{P A}\right)$, where $T S_{P A}$ is a time stamp, $b_{1}=w_{1}^{r_{k}}, b_{2}=g^{r_{k} \prod_{j=1}^{n} H_{1}\left(P I D_{M N_{j}}\right)}, b_{3}=g^{\alpha_{k}}$ and $b_{4}=d_{3} \cdot g_{1}^{\alpha_{k} \cdot H_{1}\left(P A, \Sigma_{M N}, b_{1}, b_{2}, b_{3}, k, T S_{P A}\right)}$. The adversary cannot recover the random number $r_{k}$ from $b_{1}$ or $b_{2}$ due to the intractability of discrete logarithmic problem (DLP). Then, the adversary cannot deduce the IoT key $k$ from the key extraction auxiliary message $K E A$. Thus, the confidentiality of IoT key is ensured.

\subsection{Authentication of IoT key distribution}

In the authenticated e-health IoT key extraction algorithm (shown in Subsection 4.6), the medical node $M N$ in patient $P A$ 's IoT network utilizes the element $f_{2}=g_{1}^{\frac{1}{\alpha_{P A}-H_{1}(P I D M N)}}$ in its secret key $S K_{M N}$ to recover the IoT key $k=H_{0}\left[e\left(b_{1} \cdot b_{2}^{1 / \prod_{j=1, j \neq i}^{n} H_{1}\left(P I D_{M N_{j}}\right)}, f_{2}\right)\right]$. Then, $M N$ authenticates that whether the received key extraction auxiliary message $K E A$ is sent from $P A$ by calculating

$$
e\left(g, b_{4}\right)=e\left(g_{1}, g_{2} \cdot\left(w_{P A, 2}\right)^{H_{1}\left(P I D_{P A}\right)} \cdot b_{3}^{H_{1}\left(P A, \Sigma_{M N}, b_{1}, b_{2}, b_{3}, k, T S_{P A}\right)}\right) .
$$

$K E A$ is authenticated to be a valid key extraction auxiliary message from $P A$ when the above equation holds.

Since $b_{4}=d_{P A, 3} \cdot g_{1}^{\alpha_{k} \cdot H_{1}\left(P A, \Sigma_{M N}, b_{1}, b_{2}, b_{3}, k, T S_{P A}\right)}, d_{P A, 3}=g_{1}^{\beta+\beta_{P A} \cdot H_{1}\left(P I D_{P A}\right)}$,

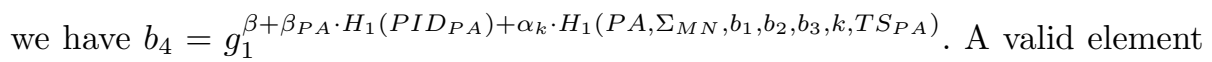
$b_{4}$ cannot be constructed without $\beta_{P A}$, which is an element in $P A$ 's secret key $S K_{P A}$. In the above verification equation, an element $w_{P A, 2}$ of $P A$ 's public 
key $P K_{P A}$ is utilized to verify whether $b_{4}$ contains the element $\beta_{P A}$. Thus, a valid $K E A$ cannot be generated without $P A$ 's secret key $S K_{P A}$ and the authentication of IoT key distribution is realized.

\subsection{Confidentiality of IoT message}

In the IoT message encryption algorithm (shown in Subsection 4.7), the ciphertext of IoT message is $C_{m}=\left(\Phi_{m, 0}, \Phi_{m, 1}, \Phi_{m, 2}, T S_{M N}\right)$, where $\Phi_{m, 0}=$ $\operatorname{SEnc}(m, k), \Phi_{m, 1}=g^{r_{m}}, \Phi_{m, 2}=f_{1} \cdot g_{1}^{r_{m} \cdot H_{1}\left(P I D_{P A}, P I D_{M N}, \Phi_{m, 0}, T S_{M N}\right)}$ and $T S_{M N}$ is a time stamp. Since the IoT message $m$ is encrypted to $\Phi_{m, 0}=$ $\operatorname{SEnc}(m, k)$, the confidentiality of IoT message is guaranteed by the confidentiality of the IoT key $k$ and the security of SEnc algorithm.

\subsection{Authentication of IoT message}

In the e-health IoT message verification and decryption algorithm (shown in Subsection 4.8), the source of IoT ciphertext is authenticated by the equation

$$
\begin{aligned}
& e\left(g, \Phi_{m, 2}\right) \\
= & e\left[g_{1}, w_{P A, 1} \cdot\left(P K_{M N}\right)^{H_{1}\left(P I D_{P A}, P I D_{M N}\right)} \cdot\left(\Phi_{m, 1}\right)^{H_{1}\left(P I D_{P A}, P I D_{M N}, \Phi_{m, 0}, T S_{M N}\right)}\right] .
\end{aligned}
$$

Since the IoT ciphertext element $\Phi_{m, 2}=f_{1} \cdot g_{1}^{r_{m} \cdot H_{1}\left(P I D_{P A}, P I D_{M N}, \Phi_{m, 0}, T S_{M N}\right)}$ and $f_{1}=g_{1}^{\alpha_{P A}+\alpha_{M N} \cdot H_{1}\left(P I D_{P A}, P I D_{M N}\right)}$ is an element in $M N$ 's secret key $S K_{M N}$, $\Phi_{m, 2}$ cannot be constructed without $f_{1}$. In the above verification equation, $M N$ 's public key $P K_{M N}=g^{\alpha_{M N}}$ is utilized to verify whether $\Phi_{m, 2}$ contains the element $\alpha_{M N}$. Thus, a valid IoT ciphertext $C_{m}$ cannot be generated without $M N$ 's secret key $S K_{M N}$ and the authentication of IoT message is realized.

\subsection{Secure against replay attack}

In the e-health IoT key distribution procedure (includes e-health IoT key generation algorithm and authenticated e-health IoT key extraction algorithm), a time stamp $T S_{P A}$ is selected by the patient $P A$ to indicate the IoT key generation time. The time stamp $T S_{P A}$ is embedded in the key extraction auxiliary message $K E A$ by calculating $b_{4}=d_{P A, 3} \cdot g_{1}^{\alpha_{k} \cdot H_{1}\left(P A, \Sigma_{M N}, b_{1}, b_{2}, b_{3}, k, T S_{P A}\right)}$, and 
verified in the authentication equation $e\left(g, b_{4}\right)=e\left(g_{1}, g_{2} \cdot\left(w_{P A, 2}\right)^{H_{1}\left(P I D_{P A}\right)}\right.$.

$\left.b_{3}^{H_{1}\left(P A, \Sigma_{M N}, b_{1}, b_{2}, b_{3}, k, T S_{P A}\right)}\right)$. If the adversary replays the key extraction auxiliary message $K E A$ utilizing the original time stamp, the medical node $M N$ will discover the replay attack by checking the history time stamps. If the adversary replays the key extraction auxiliary message $K E A$ utilizing a forged time stamp, the authentication equation will not hold and the replay attack will be discovered too.

In the e-health IoT message encryption algorithm, a time stamp $T S_{M N}$ is selected by the medical node $M N$ to indicate the encryption time. The time stamp $T S_{M N}$ is embedded in the IoT ciphertext by calculating $\Phi_{m, 2}=$ $f_{1} \cdot g_{1}^{r_{m} \cdot H_{1}\left(P I D_{P A}, P I D_{M N}, \Phi_{m, 0}, T S_{M N}\right)}$, and verified in the authentication equation

$$
\begin{aligned}
& e\left(g, \Phi_{m, 2}\right) \\
= & e\left[g_{1}, w_{P A, 1} \cdot\left(P K_{M N}\right)^{H_{1}\left(P I D_{P A}, P I D_{M N}\right)} \cdot\left(\Phi_{m, 1}\right)^{H_{1}\left(P I D_{P A}, P I D_{M N}, \Phi_{m, 0}, T S_{M N}\right)}\right] .
\end{aligned}
$$

If the adversary replays the IoT ciphertext $C_{m}$ utilizing the original time stamp, the patient $P A$ will discover the replay attack by checking the history time stamps. If the adversary replays the IoT ciphertext $C_{m}$ utilizing a forged time stamp, the authentication equation will not hold and the replay attack will be discovered too.

Thus, this system is secure against replay attack.

\section{Performance Analysis and Comparison}

In this section, we firstly compare the proposed fusion of IoT and big data system with the state-of-the art schemes [31, 32, 33, 34, 35, 36] that support fine-grained access control mechanism. Then, they are evaluated using the simulation in terms of communication and computation costs.

\subsection{Comparison}

The function, communication and computation overheads of the schemes in [31, 32, 33, 34, 35, 36] and our fusion of IoT and big data system are compared. The notations in Table 2 are used in the comparison. 
Table 2: The Performance Notations

\begin{tabular}{ll}
\hline Notation & Description \\
\hline$e_{1}$ & Exponential operation in $G$ \\
$e_{2}$ & Exponential operation in $G_{T}$ \\
$e_{p}$ & Elliptic curve bilinear pairing operation \\
$|G|$ & The size of element in group $G$ \\
$\left|G_{T}\right|$ & The size of element in group $G_{T}$ \\
$\left|Z_{p}\right|$ & The size of element in $Z_{p}$ \\
$|A t t|$ & The number of total attributes in the system \\
$|S|$ & The number of attributes in attribute set $S$ \\
$n_{1}$ & The row number of matrix $\mathbb{A}$ \\
$n_{1}^{\prime}$ & The row number of matrix $\mathbb{A}^{\prime} p$ \\
$l_{1}$ & The number of the keywords extracted from the file \\
$l_{2}$ & The number of the query keywords \\
$\tau$ & The number of IoT messages in the batch verification \\
\hline
\end{tabular}




\subsubsection{Function Comparison}

The function of the schemes in 31, 32, 33, 34, 35, 36 and our fusion of e-health IoT and big data scheme is compared in Table 3.

- Anonymity and traceability: The proposed system realizes the anonymity and traceability of patient and medical nodes. The other schemes [31, 32, 33, 34, 35, 36] do not take this important issue into consideration.

- IoT message encryption: The medical data collected by the medical nodes are encrypted using the IoT key and then transmitted to the patient in our system, which protects the confidentiality of the patient's vital signs. This function is not supported by the other schemes [31, 32, 33, 34, 35, 36.

- IoT message authentication: The source authentication of IoT message is realized our system, which effectively prevents the impersonation attack. The other schemes [31, 32, 33, 34, 35, 36, do not realize this function.

- Authenticated IoT key distribution: In the IoT key distribution phase, the medical nodes are capable to authenticate whether the IoT key is sent by the patient. The scheme in [36] and our system are able to realize this function, while the other schemes [31, 32, 33, 34, 35] cannot support authenticated IoT key distribution.

- Policy update: The schemes in [31, 32, 33] and our system realize dynamic policy updating for the outsourced stored data, while the other schemes [35, 36] do not realize this function.

- Keyword match based update: In the medical big data system, the patients may have a large amount of encrypted EHRs that are encrypted using the same access policy. This proposed system enables the patient to update only part of these EHRs using the keyword match mechanism, while all the other schemes [31, 32, 33, 34, 35] do not consider this problem. 
- Keyword match: The keyword match function is studied in the [34, 35] to construction searchable encryption schemes. Our system utilizes the keyword match mechanism to control the dynamic access policy update. The other schemes [31, 32, 33] do not support keyword match function.

- Flexible subset match: The scheme in 34 only realizes single keyword search and that in [35] supports conjunctive keyword search. Flexible subset match function is realized in our system such that the encrypted EHR is deemed as match file if the query keyword set is a subset of the pre-defined keyword set in the EHR.

\subsubsection{Communication Overhead Comparison}

Table 4 shows the communication overhead of our secure fusion system. The sizes of system public parameter $P P$ and master secret key $M S K$ are constants. Thus, our scheme is a large universe construction and supports arbitrary number of attributes. The patient's (or data user) public key $P K_{P A}$ (or $P K_{U}$ ) consists of three elements in group $G$ and secret key $S K_{P A}$ (or $S K_{U}$ ) has size linearly grow with the attribute size $|S|$. The medical node's public/secret keys $P K_{M N} / S K_{M N}$ consist of one element and two element in group $G$, respectively. The size of IoT key extraction auxiliary message $K E A$ is $4|G|$ and that of $C_{m}$ is $2|G|$. The size of $C T$ linearly grow with $n_{1}$ and $l_{1}$, and the size of the policy update query $P U Q$ linearly grow with $n_{1}^{\prime}$ and $l_{2}$.

In Table 5 the sizes of public parameter, user's (or patient) attribute secret key and (EHR) file ciphertext are compared. (1) The schemes in [31, 32, 33, 35, 36] and our system all have constant public parameter size. However, $|P P|$ of the scheme 34] depends on the total attribute size $|A t t r|$ in the system. (2) The sizes of attribute secret key in schemes [31, 32, 33, 34, 35, 36] and our system increase with $|S|$. The schemes in [32, 34, 35, 36] has $|S K|$ larger than our system. (3) The size of (EHR) file ciphertext schemes [31, 32, 33, 34, 35, 36. and our system increases on the row number $n_{1}$ of the matrix $\mathbb{A}$ in the access policy. $C T$ in our system has $\left(n_{1}+l_{1}\right)$ elements in $Z_{p}$, two elements in $G$ and 
Table 3: Function Comparison

\begin{tabular}{|c|c|c|c|c|c|c|c|}
\hline Scheme & {$[31]$} & {$[32$} & {$[33$} & {$[34$} & {$[35$} & {$[36]$} & Ours \\
\hline F1 & $\times$ & $\times$ & $\times$ & $\times$ & $\times$ & $\times$ & $\sqrt{ }$ \\
\hline F2 & $\times$ & $\times$ & $\times$ & $\times$ & $\times$ & $\times$ & $\sqrt{ }$ \\
\hline F3 & $\times$ & $\times$ & $\times$ & $\times$ & $\times$ & $\times$ & $\sqrt{ }$ \\
\hline F4 & $\times$ & $\times$ & $\times$ & $\times$ & $\times$ & $\times$ & $\sqrt{ }$ \\
\hline F5 & $\times$ & $\times$ & $\times$ & $\times$ & $\times$ & $\times$ & $\sqrt{ }$ \\
\hline F6 & $\times$ & $\times$ & $\times$ & $\times$ & $\times$ & $\sqrt{ }$ & $\sqrt{ }$ \\
\hline F7 & $\sqrt{ }$ & $\sqrt{ }$ & $\sqrt{ }$ & $\times$ & $\times$ & $\times$ & $\sqrt{ }$ \\
\hline F8 & $\times$ & $\times$ & $\times$ & $\times$ & $\times$ & $\times$ & $\sqrt{ }$ \\
\hline F9 & $\times$ & $\times$ & $\times$ & $\sqrt{ }$ & $\sqrt{ }$ & $\times$ & $\sqrt{ }$ \\
\hline F10 & $\times$ & $\times$ & $\times$ & $\times$ & $\times$ & $\times$ & $\sqrt{ }$ \\
\hline
\end{tabular}

F1: patient anonymity

$F 2$ : patient traceability

F3: medical node anonymity and traceability

F4: IoT message encryption

F5: IoT message authentication

F6: authenticated IoT key distribution

F7: policy update

F8: keyword match based update

F9: keyword match

F10: flexible subset match 
Table 4: Communication Overhead of Our System

\begin{tabular}{|l|c|}
\hline Parameter & Communication Overhead \\
\hline$|P P|$ & $3|G|+\left|G_{T}\right|$ \\
\hline$|M S K|$ & $3\left|Z_{p}\right|$ \\
\hline$\left|P K_{P A}\right|$ & $3|G|$ \\
\hline$\left|S K_{P A}\right|$ & $2\left|Z_{p}\right|+(|S|+2)|G|$ \\
\hline$\left|P K_{U}\right|$ & $3|G|$ \\
\hline$\left|S K_{U}\right|$ & $2\left|Z_{p}\right|+(|S|+2)|G|$ \\
\hline$\left|P K_{M N}\right|$ & $|G|$ \\
\hline$\left|S K_{M N}\right|$ & $2|G|$ \\
\hline$|K E A|$ & $4|G|$ \\
\hline$\left|C_{m}\right|$ & $2|G|$ \\
\hline$|C T|$ & $\left(n_{1}+l_{1}\right)\left|Z_{p}\right|+2|G|+\left|G_{T}\right|$ \\
\hline$|P U Q|$ & $\left(n_{1}^{\prime}+l_{2}+1\right)\left|Z_{p}\right|$ \\
\hline
\end{tabular}

one elements in $G_{T}$. $C T$ in scheme [35] linearly grow with $l_{1}$ and $|A t t r|$, which is larger than the other schemes [31, 32, 33, 34, 36] and our system.

In Table 6, the size of access policy update query $P U Q$ in our system is compared that in schemes [31, 32, 33] and the size of keyword index is compared with that in [34, 35]. (1) The schemes [31, 32, 33] have similar policy update mechanism, where the new access policy is compared with the previous policy. The attributes in the new access policy are classified into three types, and the numbers of the the three types of attributes are denoted as $n_{11}^{\prime}, n_{12}^{\prime}, n_{13}^{\prime}$ such that $n_{11}^{\prime}+n_{12}^{\prime}+n_{13}^{\prime}=n_{1}^{\prime}$, where $n_{1}^{\prime}$ is the row number of $\mathbb{A}$. In order to simplify the comparison, we set $n_{11}^{\prime}=n_{12}^{\prime}=n_{13}^{\prime}=n_{1}^{\prime} / 3$. Since the element bit length in $Z_{p}$ is less than that in $G$, the size of access policy update query in our system is less than that in [31, 32, 33. (2) The sizes of keyword index in schemes [34, 35] are $\left(2 l_{1}+2|S|+1\right)|G|$ and $\left(l_{1}+2|S|+2\right)|G|$, respectively. That in in our system is $\left(l_{1}\right)\left|Z_{p}\right|+|G|$, which is less that the other schemes [34, 35]. 
Table 5: Communication Overhead Comparison 1

\begin{tabular}{|c|c|c|c|}
\hline Scheme & Z1 & Z2 & $\mathrm{Z3}$ \\
\hline $\operatorname{KanY} 31$ & $|G|$ & $(|S|) \cdot|G|$ & $\begin{array}{c}\left(2 n_{1}\right)|G| \\
+\left(n_{1}+1\right)\left|G_{T}\right|\end{array}$ \\
\hline Hongwei 32 & $2|G|+2\left|G_{T}\right|$ & $(2|S|) \cdot|G|$ & $\begin{array}{c}\left(2 n_{1}+1\right)|G| \\
+\left(n_{1}+1\right)\left|G_{T}\right|\end{array}$ \\
\hline Ying 33. & $2|G|$ & $(|S|) \cdot|G|$ & $\begin{array}{c}\left(2 n_{1}+1\right)|G| \\
+\left|G_{T}\right|\end{array}$ \\
\hline Jiguo 34 & $(|A t t|+4)|G|$ & $(2|S|)|G|$ & $\begin{array}{c}\left(n_{1}+2\right)|G| \\
\quad+\left|G_{T}\right|\end{array}$ \\
\hline Miao 35] & $5|G|+\left|G_{T}\right|$ & $(2|S|+2)|G|$ & $\left(n_{1}+2|A t t|+2\right)|G|$ \\
\hline Yeh 36. & $|G|+\left|G_{T}\right|$ & $(2|S|+1)|G|$ & $\begin{array}{c}\left(2 n_{1}+1\right)|G| \\
+\left|G_{T}\right|\end{array}$ \\
\hline Ours & $3|G|+\left|G_{T}\right|$ & $\begin{array}{c}2\left|Z_{p}\right| \\
+(|S|+2)|G|\end{array}$ & $\begin{array}{l}\left(n_{1}+l_{1}\right)\left|Z_{p}\right| \\
+2|G|+\left|G_{T}\right|\end{array}$ \\
\hline
\end{tabular}

Z1: size of public parameter $|P P|$

$Z 2:$ size of user's (or patient) attribute secret key $\left|S K_{U}\right|\left(\right.$ or $\left.\left|S K_{P A}\right|\right)$

Z3: size of (EHR) file ciphertext $|C T|$ 
Table 6: Communication Overhead Comparison 2

\begin{tabular}{|c|c|c|}
\hline Scheme & Z4 & Z5 \\
\hline KanY [31] & $\left(2 n_{1}^{\prime}+n_{13}^{\prime}\right)|G|$ & $\perp$ \\
\hline Hongwei [32] & $\left(2 n_{1}^{\prime}+n_{13}^{\prime}\right)|G|$ & $\perp$ \\
\hline Ying [33] & $\left(n_{1}^{\prime}+n_{13}^{\prime}\right)|G|$ & $\perp$ \\
\hline Jiguo [34] & $\perp$ & $\left(2 l_{1}+2|S|+1\right)|G|$ \\
\hline Miao [35] & $\perp$ & $\left(l_{1}+2|S|+2\right)|G|$ \\
\hline Ours & $\left(n_{1}^{\prime}+l_{2}+1\right)\left|Z_{p}\right|$ & $\left(l_{1}\right)\left|Z_{p}\right|+|G|$ \\
\hline
\end{tabular}

Z4: size of access policy update query $|P U Q|$

$Z 5$ : size of keyword index

\subsubsection{Computation Overhead Comparison}

Table 7 shows the communication overhead of our system. The global setup algorithm requires one exponentiation operation in $G$, one exponentiation operation in $G_{T}$ and one bilinear pairing calculation. The computation overheads of patient, user and medical node registration algorithms are $(|S|+5) e_{1},(|S|+5) e_{1}$ and $3 e_{1}$, respectively. The IoT key generation algorithm consumes 4 exponentiation operations in $G$, and IoT key extraction algorithm needs 3 exponentiation operations in $G$ and 3 bilinear pairing calculation. The computation overheads of IoT message encryption algorithm, IoT message verification and decryption algorithm, and IoT message batch verification and decryption algorithm are $2 e_{1}$, $2 e_{1}+2 e_{p}$ and $(3 \tau) e_{1}+2 e_{p}$, respectively, where $\tau$ is the number of IoT messages in the batch verification. The EHR file encryption algorithm consumes two exponentiation operation in $G$ and one exponentiation operation in $G_{T}$. The policy update query generation algorithm does not require any exponentiation or bilinear paring calculation, which is denoted as " $\approx 0$ ". The computation overheads of ciphertext update algorithm and EHR decryption algorithms are $e_{2}$ and $\left(n_{1}\right) e_{1}+e_{p}$, respectively.

In Table 8, our system is compared with the schemes in [31, 32, 33, 34, 35, 36. 
Table 7: Computation Overhead of Our System

\begin{tabular}{|c|c|l|c|}
\hline \multicolumn{2}{|l|}{ Algorithm } & Computation Overhead \\
\hline Alg. & 1 & Global Setup & $e_{1}+e_{2}+e_{p}$ \\
\hline Alg. & 2 & Patient Reg. & $(|S|+5) e_{1}$ \\
\hline Alg. & 3 & User Reg. & $(|S|+5) e_{1}$ \\
\hline Alg. & 4 & Medical Node Reg. & $3 e_{1}$ \\
\hline Alg. & 5 & IoT Key Gen. & $4 e_{1}$ \\
\hline Alg. & 6 & IoT Key Extraction & $3 e_{1}+3 e_{p}$ \\
\hline Alg. & 7 & IoT Msg. Enc. & $2 e_{1}$ \\
\hline Alg. & 8 & IoT Msg. Ver. \& Dec & $2 e_{1}+2 e_{p}$ \\
\hline Alg. & 9 & IoT Bat. Ver. \& Dec & $(3 \tau) e_{1}+2 e_{p}$ \\
\hline Alg. & 10 & EHR Enc. & $2 e_{1}+e_{2}$ \\
\hline Alg. & 12 & Policy Update Query & $\approx 0$ \\
\hline Alg. & 12 & Ciphertext Update & \\
\hline Alg. & 11 & Dec & $e_{2}$ \\
\hline
\end{tabular}


(1) The computation costs for user (or patient) attribute secret key of these schemes linearly increase with $|S|$. Our system requires $|S|+2$ exponentiation operations in group $G$ to generate an attribute secret key, which is smaller than that in the other schemes [31, 32, 33, 34, 35, 36. (2) The computation costs for (EHR) file encryption in schemes [31, 32, 33, 34, 35, 36] linearly grow with the parameter $n_{1}$, which is the row number of matrix $\mathbb{A}$ in the access policy. Our system requires two exponentiation operations in group $G$ and one exponentiation operations in group $G_{T}$ to generate a EHR ciphertext, which is much smaller than that in the other schemes [31, 32, 33, 34, 35, 36]. (3) The computation costs for (EHR) file decryption also depends on $n_{1}$. Our system requires $n_{1}$ exponentiation operations in group $G$ and two bilinear pairing operations to decrypt a EHR ciphertext, which is smaller than that in [31, 32, 33, 35, 36] and larger than that in 34 .

Table 9 shows the computation cost for access policy update query generation algorithm and ciphertext policy update algorithm. (1) The schemes in 31 , 32, 33. require $\left(2 n_{1}^{\prime}+3 n_{13}^{\prime}\right) e_{1},\left(2 n_{1}^{\prime}+3 n_{13}^{\prime}\right) e_{1}$ and $\left(n_{1}^{\prime}+2 n_{13}^{\prime}\right) e_{1}$ to generate $P U Q$, while our system does not need any exponentiation or bilinear pairing calculation. (2) In the ciphertext policy update phase, our system consumes one exponentiation operation in group $G_{T}$, which is much smaller than the computation costs in other schemes [31, 32, 33].

Table 10 shows the computation cost for keyword index generation and keyword match test. (1) The schemes in [34, 35] require $e_{1}$ and $\left(2 l_{2}\right) e_{p}$ to generate encrypted keyword index, while the computation cost of that in our system is one exponentiation operation in group $G$. (2) In the keyword match test phase, our system consumes one exponentiation operation in group $G_{T}$, which is much smaller than the computation costs in other schemes [34, 35].

\subsection{Simulation}

The performance of this proposed system and the other schemes in [31, 32, 33, 34, 35, 36] are evaluated by the simulations, which are implemented on a personal computer running Windows 10 and 64-bit operation system with 
Table 8: Computation Overhead Comparison 1

\begin{tabular}{|c|c|c|c|}
\hline Scheme & T1 & T2 & T3 \\
\hline KanY [31] & $(2|S|) e_{1}$ & $\begin{array}{c}\left(2 n_{1}\right) e_{1}+e_{p} \\
+\left(2 n_{1}+1\right) e_{2}\end{array}$ & $\begin{array}{c}\left(3 n_{1}\right) e_{2} \\
+\left(3 n_{1}\right) e_{p}\end{array}$ \\
\hline Hongwei [32] & $(3|S|) e_{1}$ & $\begin{array}{c}\left(3 n_{1}+1\right) e_{1} \\
+\left(2 n_{1}+1\right) e_{2}+e_{p}\end{array}$ & $\begin{array}{c}\left(2 n_{1}\right) e_{1}+\left(n_{1}\right) e_{2} \\
+\left(3 n_{1}\right) e_{p}\end{array}$ \\
\hline Ying [33] & $\begin{array}{c}(|S|) e_{1} \\
+(2|S|) e_{p}\end{array}$ & $\begin{array}{c}\left(3 n_{1}+1\right) e_{1} \\
+\left(2 n_{1}+1\right) e_{2}+e_{p}\end{array}$ & $\begin{array}{c}\left(n_{1}\right) e_{2} \\
+\left(2 n_{1}+1\right) e_{p}\end{array}$ \\
\hline Jiguo [34] & $(3|S|) e_{1}$ & $\begin{array}{c}\left(n_{1}+2\right) e_{1} \\
+e_{2}\end{array}$ & $\begin{array}{c}2 e_{1}+3 e_{p} \\
\text { Miao [35] }\end{array}$ \\
\hline Yeh [36] & $(3|S|+2) e_{1}$ & $\left(4 n_{1}\right) e_{1}$ & $\left(n_{1}\right) e_{2}+\left(n_{1}\right) e_{p}$ \\
\hline Ours & $(|S|+2) e_{1}$ & $2 e_{1}+e_{2}$ & $n_{1} \cdot e_{1}+2 e_{p}$ \\
\hline
\end{tabular}

T1: computation cost for user (or patient) attribute secret key

T2: computation cost for (EHR) file encryption

T3: computation cost for (EHR) file decryption

Table 9: Computation Overhead Comparison 2

\begin{tabular}{|c|c|c|}
\hline Scheme & T4 & T5 \\
\hline KanY [31] & $\left(2 n_{1}^{\prime}+3 n_{13}^{\prime}\right) e_{1}$ & $\left(n_{13}^{\prime}\right) e_{p}$ \\
\hline Hongwei [32] & $\left(2 n_{1}^{\prime}+3 n_{13}^{\prime}\right) e_{1}$ & $\left(2 n_{12}^{\prime}\right) e_{1}+\left(n_{12}^{\prime}\right) e_{2}+\left(n_{1}^{\prime}\right) e_{p}$ \\
\hline Ying [33] & $\left(n_{1}^{\prime}+2 n_{13}^{\prime}\right) e_{1}$ & $\left(2 n_{1}^{\prime}+n_{12}^{\prime}\right) e_{1}$ \\
\hline Ours & $\approx 0$ & $e_{2}$ \\
\hline
\end{tabular}

T4: computation cost for access policy update query generation T5: computation cost for ciphertext policy update 
Table 10: Computation Overhead Comparison 3

\begin{tabular}{|c|c|c|}
\hline Scheme & T6 & T7 \\
\hline Jiguo [34] & $e_{1}$ & $\left(2 l_{2}\right) e_{p}$ \\
\hline Miao [35] & $\left(3 l_{1}+2|S|+1\right) e_{1}$ & $\left(n_{1}\right) e_{2}+\left(2 n_{1}+3 l_{2}\right) e_{p}$ \\
\hline Ours & $e_{1}$ & $e_{2}$ \\
\hline
\end{tabular}

T6: computation cost for keyword index generation

T7: computation cost for keyword match test

Intel(R) Core(TM) i3-2120 CPU @ $3.30 \mathrm{GHz}, 4.00$ GB RAM. The Type-A elliptic curve $E / F_{p}: y^{2}=x^{3}+x$ over $F_{p}$ is selected to conduct experiments, and the group $G$ has order $q$, where the bit length of $p$ and $q$ are 160-bit and 512-bit, respectively. Thus, the lengths of the elements in $G, G_{T}$ and $Z_{p}$ are 1024-bit, 1024-bit and 160-bit, respectively.

\subsubsection{Transmission Efficiency}

Fig. 6 shows the transmission cost of public parameter and the value of $\mathrm{x}$ axis varies with the number of total attributes. The sizes of public parameter in schemes [31, 32, 33, 35, 36] and our system are constants, which are $0.512 \mathrm{~KB}$, $0.128 \mathrm{~KB}, 0.512 \mathrm{~KB}, 0.256 \mathrm{~KB}, 0.768 \mathrm{~KB}$ and $0.256 \mathrm{~KB}$, respectively. $|P P|$ in 34] grows with $|A t t|$, which is $3.072 \mathrm{~KB}$ when $|A t t|=20$ and $13.312 \mathrm{~KB}$ when $|A t t|=100$.

Fig. 7 shows the transmission cost of user's attribute secret key and the value of $\mathrm{x}$-axis varies with the number of attributes. When $|S|=20$, the sizes of user's attribute secret key in schemes [31, 32, 33, 34, 35, 36] and our system are $2.56 \mathrm{~KB}, 5.12 \mathrm{~KB}, 2.56 \mathrm{~KB}, 5.12 \mathrm{~KB}, 5.376 \mathrm{~KB}, 5.248 \mathrm{~KB}$ and $2.856 \mathrm{~KB}$, respectively. When $|S|=100$, the sizes of user's attribute secret key in schemes [31, 32, 33, 34, 35, 36] and our system are $12.8 \mathrm{~KB}, 25.6 \mathrm{~KB}, 12.8 \mathrm{~KB}, 25.6 \mathrm{~KB}$, $25.856 \mathrm{~KB}, 25.728 \mathrm{~KB}$ and $13.096 \mathrm{~KB}$, respectively. Thus, our system has good efficiency in transmitting user's attribute secret key.

Fig. 8 shows the transmission cost of $C T$ and the value of $\mathrm{x}$-axis varies 


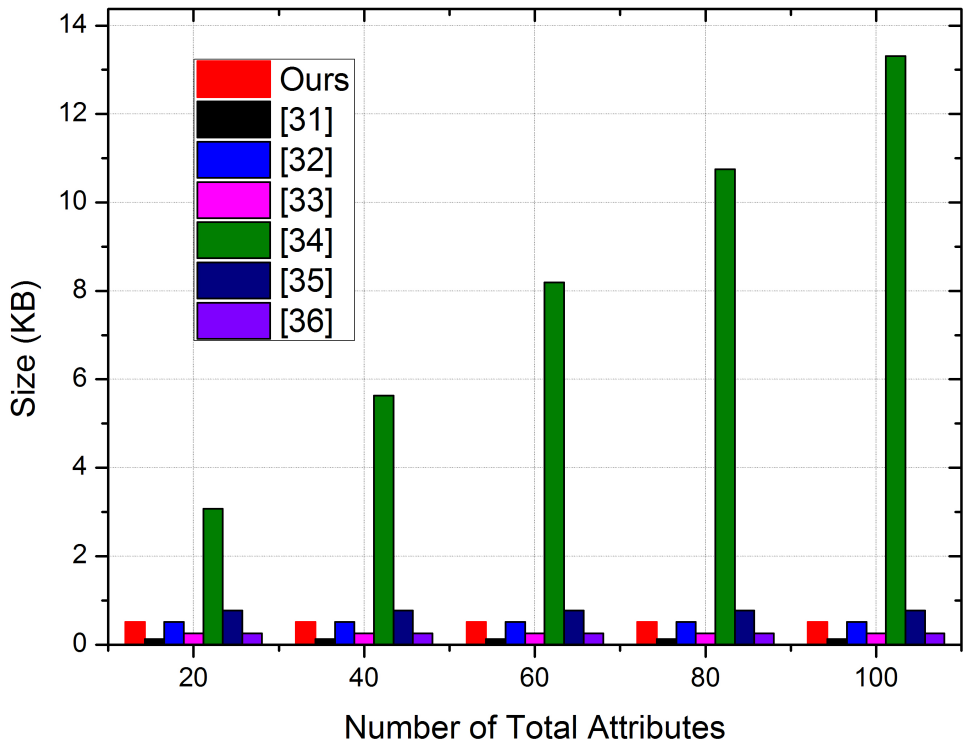

Figure 6: Transmission Cost of Public Parameter 


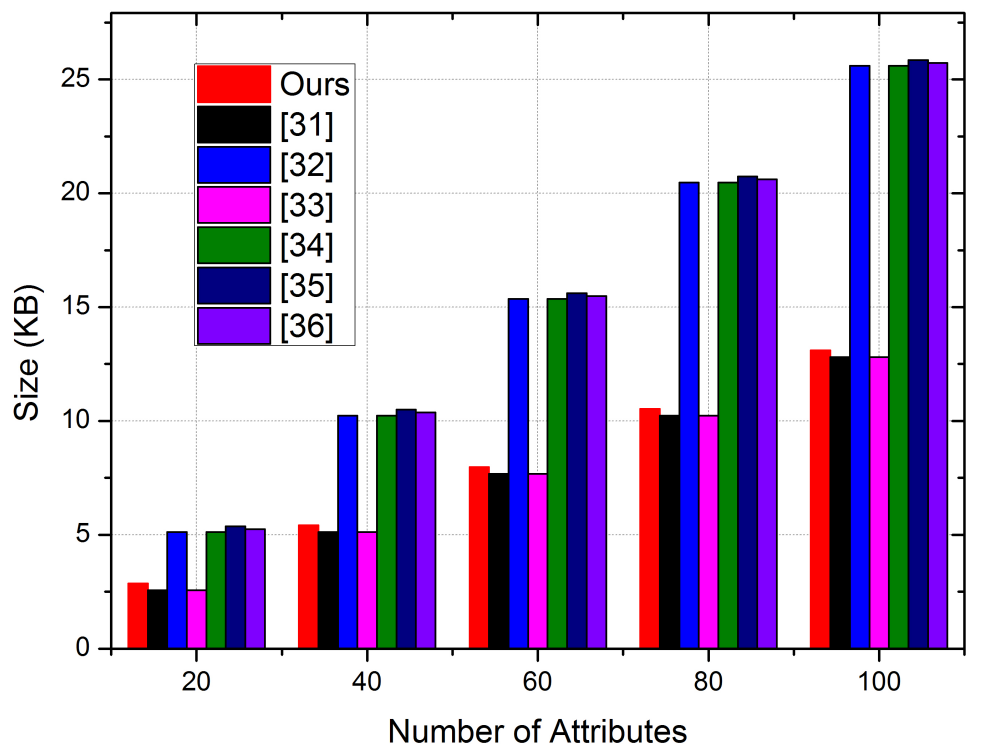

Figure 7: Transmission Cost of User's Attribute Secret Key 
with the number of attributes. When $\left|n_{1}\right|=20$, the sizes of $C T$ in schemes [31, 32, 33, 34, 35, 36] and our system are 7.808 KB, 7.936 KB, 5.376 KB, 2.944 $\mathrm{KB}, 28.416 \mathrm{~KB}, 5.376 \mathrm{~KB}$ and $1.012 \mathrm{~KB}$, respectively. When $\left|n_{1}\right|=100$, the sizes of user's attribute secret key in schemes [31, 32, 33, 34, 35, 36] and our system are $38.528 \mathrm{~KB}, 38.656 \mathrm{~KB}, 25.856 \mathrm{~KB}, 13.184 \mathrm{~KB}, 38.656 \mathrm{~KB}, 25.856$ $\mathrm{KB}$ and $2.612 \mathrm{~KB}$, respectively. It is obvious that our system has the least $|C T|$ transmission cost.

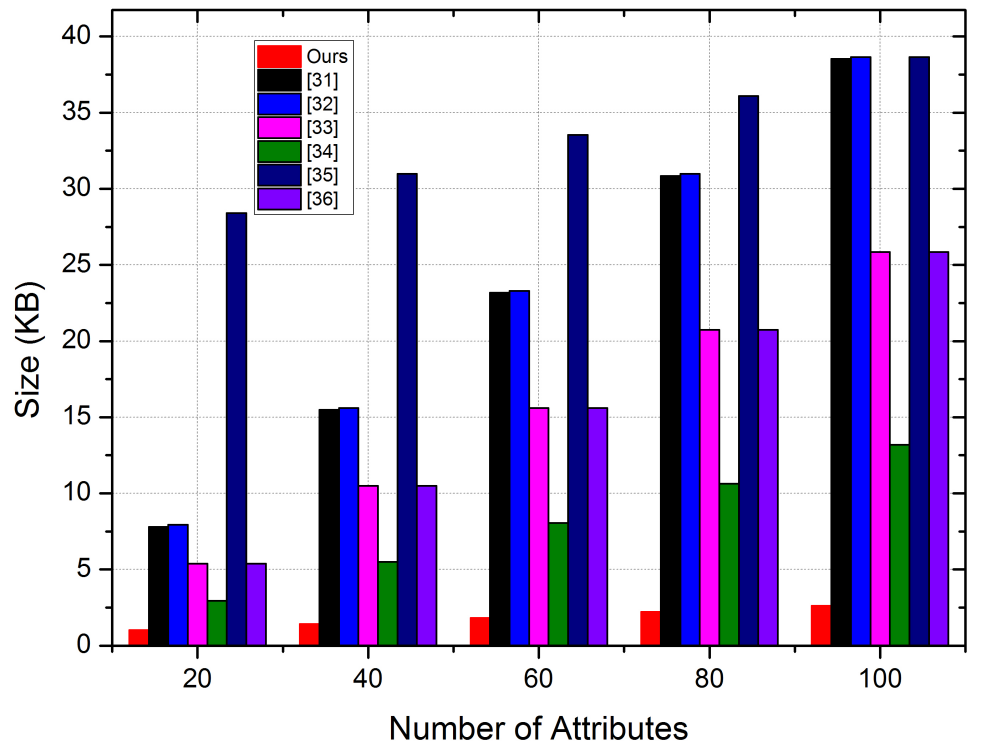

Figure 8: Transmission Cost of Ciphertext

Fig. 9 shows the transmission cost of $P U Q$ and the value of $\mathrm{x}$-axis varies with the number of attributes. When $\left|n_{1}^{\prime}\right|=15$, the sizes of $P U Q$ in schemes [31, 32, 33 and our system are $4.48 \mathrm{~KB}, 4.48 \mathrm{~KB}, 2.56 \mathrm{~KB}$ and $0.42 \mathrm{~KB}$, respectively. When $\left|n_{1}^{\prime}\right|=60$, the sizes of user's attribute secret key in schemes [31, 32, 33] and our system are 17.92 KB, $17.92 \mathrm{~KB}, 10.24 \mathrm{~KB}$ and $1.32 \mathrm{~KB}$, respectively. Our system also has the least $|P U Q|$ transmission cost. 


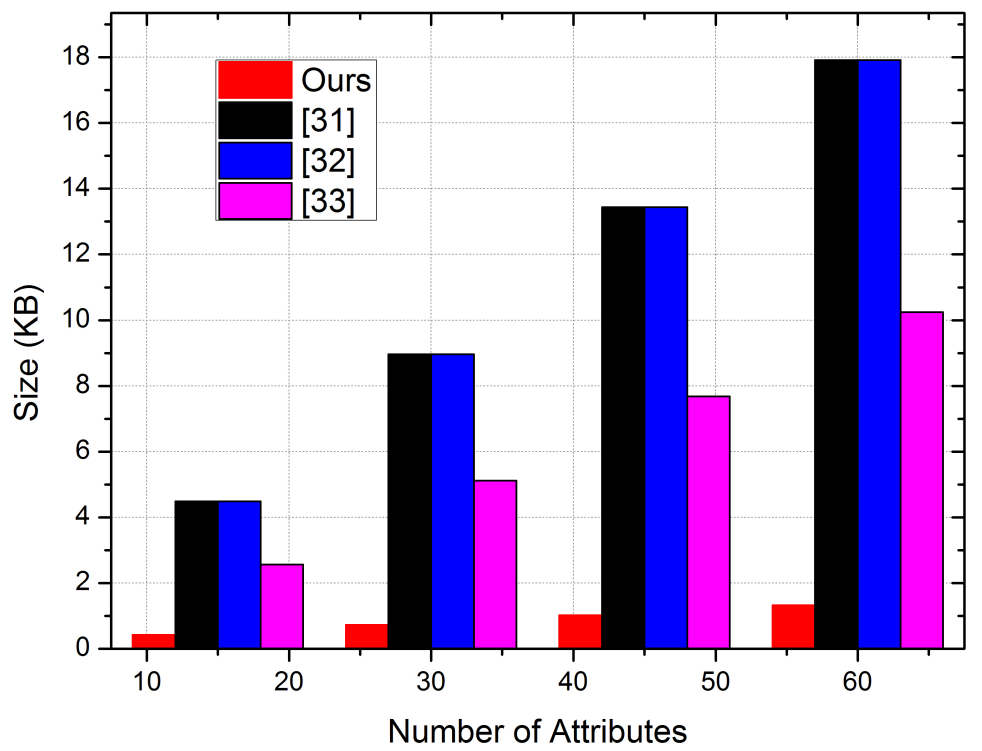

Figure 9: Transmission Cost of Policy Update Query 
Fig. 10 shows the transmission cost of keyword encrypted index and the value of $\mathrm{x}$-axis varies with the number of extracted keywords from the (EHR) file. When $\left|l_{1}\right|=5$, the sizes of keyword encrypted index in schemes [34, 35] and our system are $6.528 \mathrm{~KB}, 6.016 \mathrm{~KB}$ and $0.228 \mathrm{~KB}$, respectively. When $\left|l_{1}\right|=30$, the sizes of keyword encrypted index in schemes [34, 35] and our system are $12.928 \mathrm{~KB}, 9.216 \mathrm{~KB}$ and $0.728 \mathrm{~KB}$, respectively. Our system has the least keyword encrypted index transmission cost.

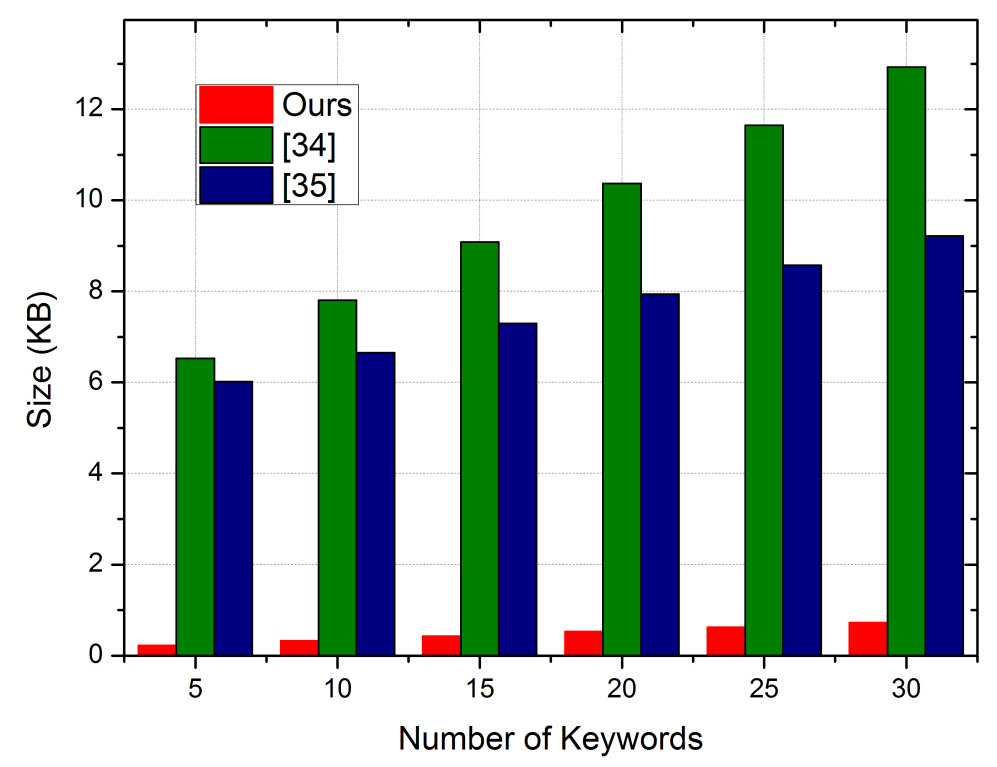

Figure 10: Transmission Cost of Keyword Encrypted Index 


\subsubsection{Computation Efficiency}

Fig. 11 shows the computation cost of user's attribute secret key generation and the value of x-axis varies with the number of user's attributes. When $|S|=$ 20 , the computation cost in schemes [31, 32, 33, 34, 35, 36] and our system are $0.368 \mathrm{~s}, 0.552 \mathrm{~s}, 0.905 \mathrm{~s}, 0.552 \mathrm{~s}, 0.57 \mathrm{~s}, 0.561 \mathrm{~s}$ and $0.202 \mathrm{~s}$, respectively. When $|S|=100$, the computation cost in schemes [31, 32, 33, 34, 35, 36] and our system are $1.839 \mathrm{~s}, 2.759 \mathrm{~s}, 4.525 \mathrm{~s}, 2.759 \mathrm{~s}, 2.777 \mathrm{~s}, 2.768 \mathrm{~s}$ and $0.938 \mathrm{~s}$, respectively. Our system has the least attribute secret key generation computation cost.

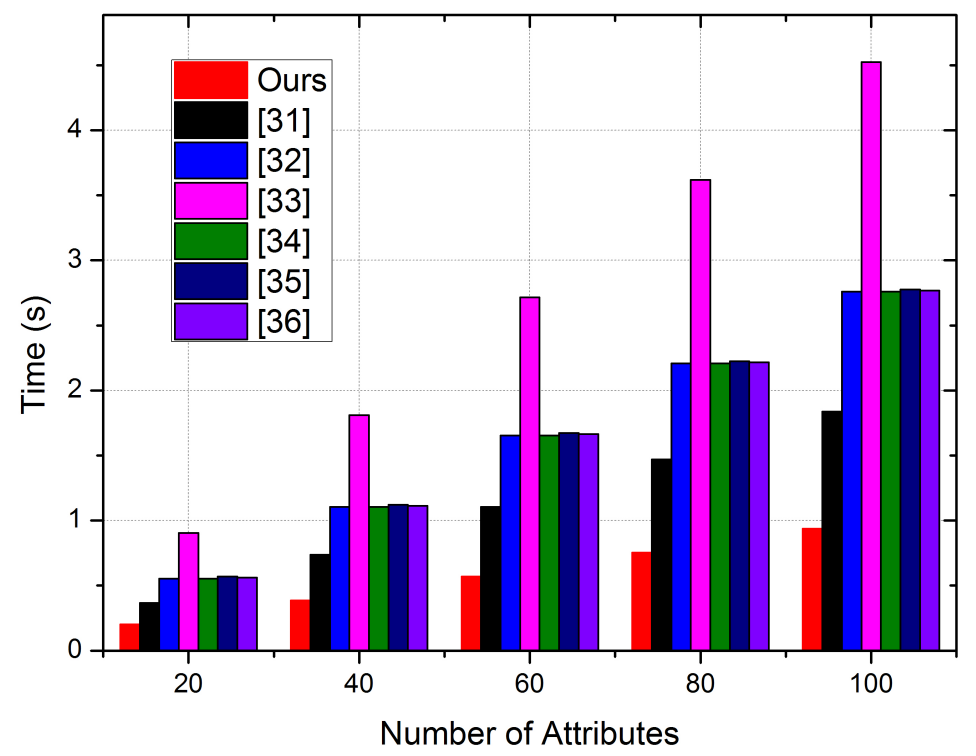

Figure 11: Computation Cost of User's Attribute Secret Key Generation

Fig. 12 and Table 11 show the computation cost of (EHR) file encryption and the value of $\mathrm{x}$-axis varies with $n_{1}$. When $\left|n_{1}\right|=20$, the computation cost in schemes [31, 32, 33, 34, 35, 36] and our system are $0.492 \mathrm{~s}, 0.689 \mathrm{~s}, 0.685 \mathrm{~s}, 0.205$ s, $0.736 \mathrm{~s}, 0.380 \mathrm{~s}$ and $0.021 \mathrm{~s}$, respectively. When $\left|n_{1}\right|=100$, the computation cost in schemes [31, 32, 33, 34, 35, 36 and our system are $2.377 \mathrm{~s}, 3.299 \mathrm{~s}, 3.305$ 
s, $0.941 \mathrm{~s}, 3.678 \mathrm{~s}, 1.851 \mathrm{~s}$ and $0.022 \mathrm{~s}$, respectively. Our system has the least (EHR) file encryption computation cost.

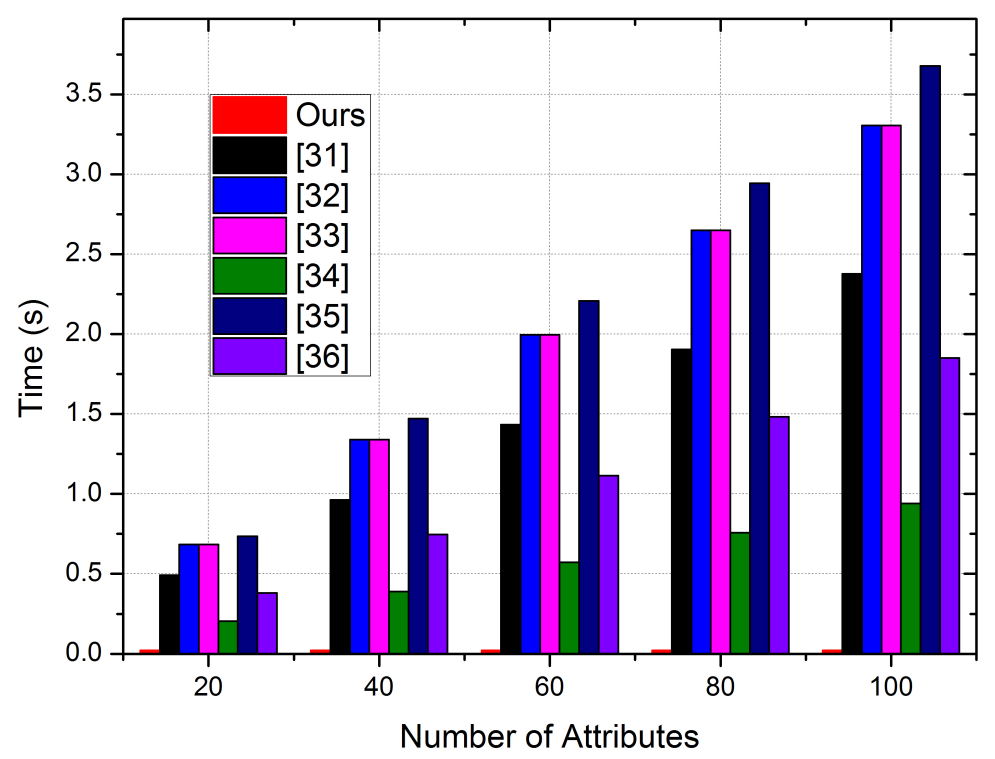

Figure 12: Computation Cost of EHR Encryption

Fig. 13 shows the computation cost of (EHR) file decryption and the value of $\mathrm{x}$-axis varies with $n_{1}$. When $\left|n_{1}\right|=20$, the computation cost in schemes [31, 32, 33, 34, 35, 36] and our system are $1.237 \mathrm{~s}, 1.501 \mathrm{~s}, 0.791 \mathrm{~s}, 0.072 \mathrm{~s}$, $0.412 \mathrm{~s}, 0.905 \mathrm{~s}$ and $0.22 \mathrm{~s}$, respectively. When $\left|n_{1}\right|=100$, the computation cost in schemes [31, 32, 33, 34, 35, 36] and our system are $6.183 \mathrm{~s}, 7.506 \mathrm{~s}, 3.882 \mathrm{~s}$, $0.072 \mathrm{~s}, 2.061 \mathrm{~s}, 4.525 \mathrm{~s}$ and $0.956 \mathrm{~s}$, respectively. Our system has (EHR) file decryption computation cost lower than that in [31, 32, 33, 35, 36] and higher than that in [34.

Fig. 14 and Table 12 show the computation cost of access policy update query generation and the value of x-axis varies with $n_{1}^{\prime}$. When $\left|n_{1}^{\prime}\right|=15$, the computation cost in schemes [31, 32, 33] and our system are $0.419 \mathrm{~s}, 0.414 \mathrm{~s}$, 
Table 11: Computation Cost of EHR Encryption

\begin{tabular}{|l|c|c|c|c|c|c|c|}
\hline $\mathbf{n}_{\mathbf{1}}$ & Ours & {$[31]$} & {$[32]$} & {$[33]$} & {$[34$} & {$[35]$} & {$[36]$} \\
\hline $\mathbf{2 0}$ & $\mathbf{0 . 0 2 1}$ & 0.492 & 0.689 & 0.685 & 0.205 & 0.736 & 0.380 \\
\hline $\mathbf{4 0}$ & $\mathbf{0 . 0 2 9}$ & 0.963 & 1.338 & 1.340 & 0.389 & 1.471 & 0.747 \\
\hline $\mathbf{6 0}$ & $\mathbf{0 . 0 2 5}$ & 1.434 & 1.988 & 1.995 & 0.573 & 2.207 & 1.115 \\
\hline $\mathbf{8 0}$ & $\mathbf{0 . 0 1 9}$ & 1.905 & 2.642 & 2.650 & 0.757 & 2.943 & 1.483 \\
\hline $\mathbf{1 0 0}$ & $\mathbf{0 . 0 2 2}$ & 2.377 & 3.299 & 3.305 & 0.941 & 3.678 & 1.851 \\
\hline
\end{tabular}

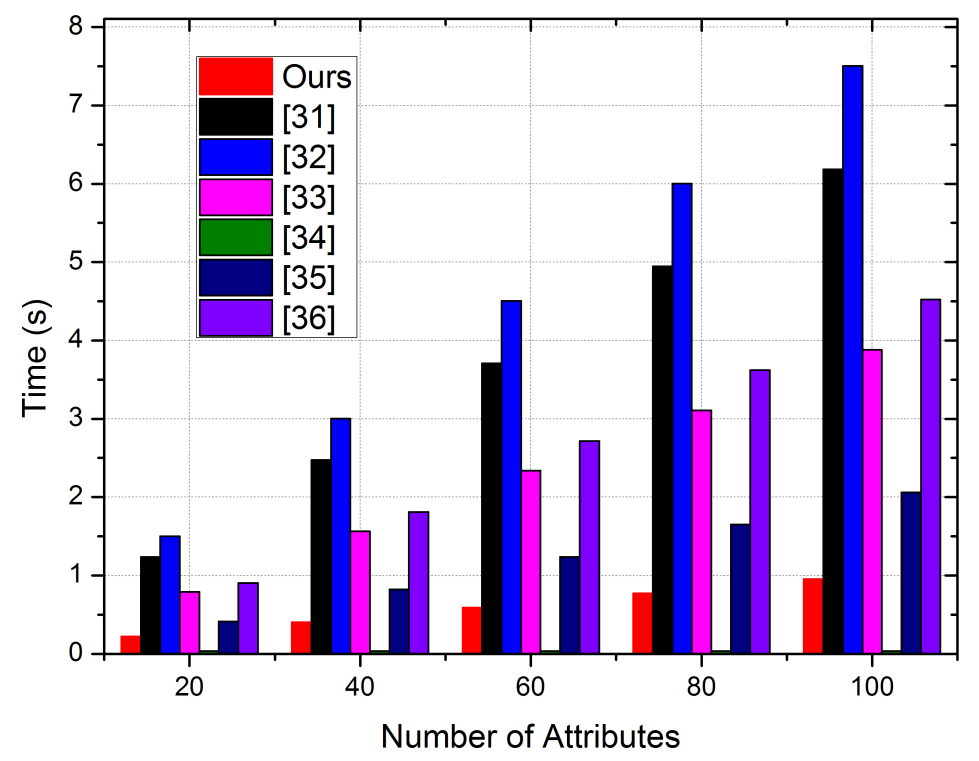

Figure 13: Computation Cost of EHR Decryption 
$0.237 \mathrm{~s}$ and $0.0009 \mathrm{~s}$, respectively. When $\left|n_{1}^{\prime}\right|=60$, the computation cost in schemes [31, 32, 33] and our system are $1.658 \mathrm{~s}, 1.655 \mathrm{~s}, 0.928 \mathrm{~s}$ and $0.0011 \mathrm{~s}$, respectively. Our system has the least access policy update query generation computation cost.

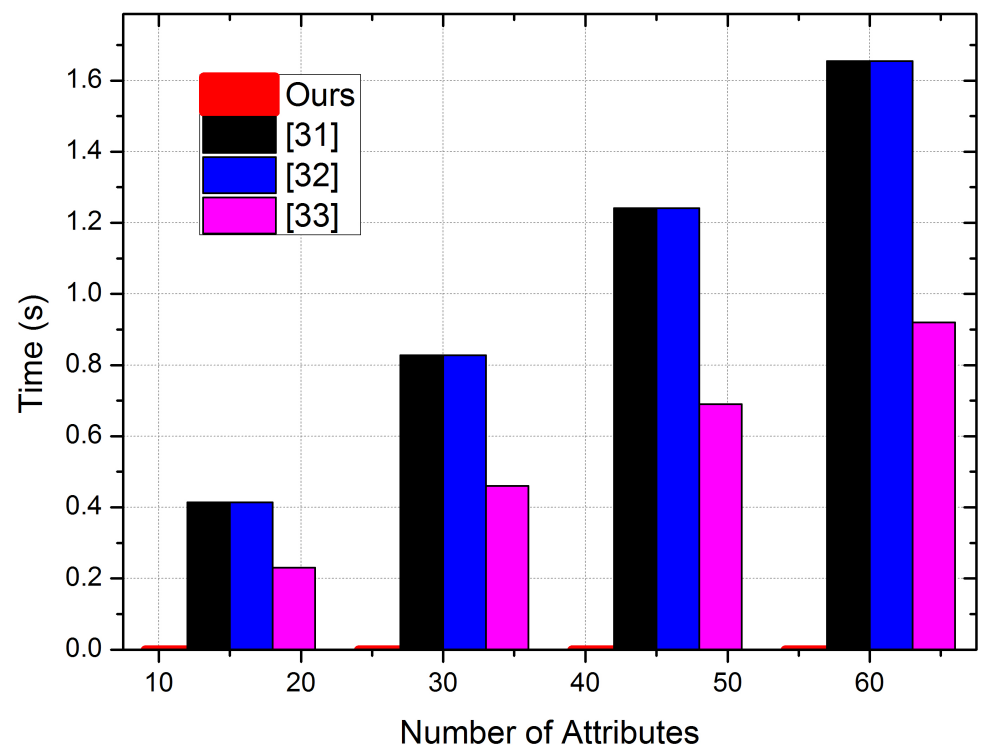

Figure 14: Computation Cost of Access Policy Update Query

Fig. 15 and Table 13 show the computation cost of ciphertext access policy update and the value of x-axis varies with $n_{1}^{\prime}$. When $\left|n_{1}^{\prime}\right|=15$, the computation cost in schemes [31, 32, 33] and our system are $0.092 \mathrm{~s}, 0.375 \mathrm{~s}, 0.322 \mathrm{~s}$ and 0.0029 $\mathrm{s}$, respectively. When $\left|n_{1}^{\prime}\right|=60$, the computation cost in schemes [31, 32, 33. and our system are $0.361 \mathrm{~s}, 1.501 \mathrm{~s}, 1.287 \mathrm{~s}$ and $0.0039 \mathrm{~s}$, respectively. Our system has the least ciphertext access policy update computation cost.

Fig. 16 and Table 14 show the computation cost of keyword index generation and the value of $\mathrm{x}$-axis varies with $l_{1}$. To simplify the comparison, the attribute size $|S|$ is set to be 10 in scheme [34]. When $\left|l_{1}\right|=5$, the computation cost in 
Table 12: Computation Cost of Access Policy Update Query

\begin{tabular}{|c|c|c|c|c|}
\hline $\mathbf{n}_{\mathbf{1}}^{\prime}$ & Ours & $31]$ & {$[32]$} & {$[33$} \\
\hline $\mathbf{1 5}$ & $\mathbf{0 . 0 0 0 9}$ & 0.419 & 0.414 & 0.237 \\
\hline $\mathbf{3 0}$ & $\mathbf{0 . 0 0 0 9}$ & 0.825 & 0.828 & 0.460 \\
\hline $\mathbf{4 5}$ & $\mathbf{0 . 0 0 1 4}$ & 1.232 & 1.241 & 0.692 \\
\hline $\mathbf{6 0}$ & $\mathbf{0 . 0 0 1 1}$ & 1.658 & 1.655 & 0.928 \\
\hline
\end{tabular}

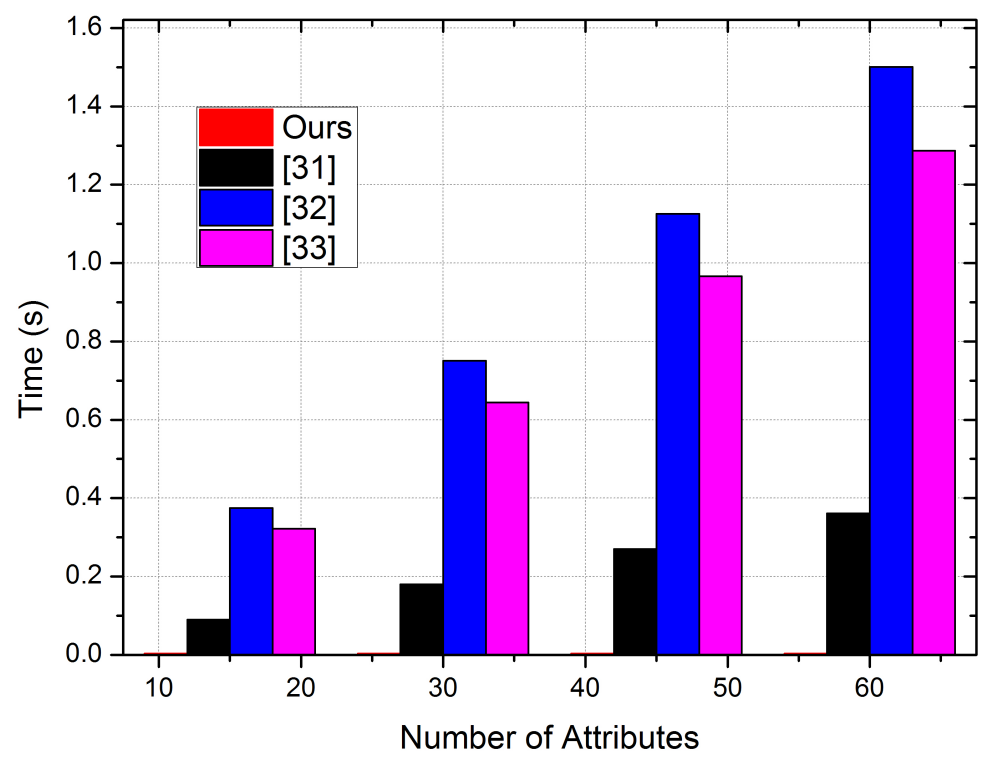

Figure 15: Computation Cost of Ciphertext Update 
Table 13: Computation Cost of Ciphertext Update

\begin{tabular}{|c|c|c|c|c|}
\hline $\mathbf{n}_{\mathbf{1}}^{\prime}$ & Ours & {$[31]$} & {$[32]$} & {$[33]$} \\
\hline $\mathbf{1 5}$ & $\mathbf{0 . 0 0 2 9}$ & 0.092 & 0.375 & 0.322 \\
\hline $\mathbf{3 0}$ & $\mathbf{0 . 0 0 3 2}$ & 0.186 & 0.751 & 0.644 \\
\hline $\mathbf{4 5}$ & $\mathbf{0 . 0 0 3 7}$ & 0.275 & 1.126 & 0.966 \\
\hline $\mathbf{6 0}$ & $\mathbf{0 . 0 0 3 9}$ & 0.361 & 1.501 & 1.287 \\
\hline
\end{tabular}

schemes [34, 35] and our system are $0.330 \mathrm{~s}, 0.0089 \mathrm{~s}$ and $0.0091 \mathrm{~s}$, respectively. When $\left|l_{1}\right|=30$, the computation cost in schemes [34, 35] and our system are $1.018 \mathrm{~s}, 0.0092 \mathrm{~s}$ and $0.0091 \mathrm{~s}$, respectively. Our system has the least keyword index generation computation cost. Although the scheme in 35 is also efficient, it can not realize flexible subset keyword search.

Table 14: Computation Cost of Keyword Index Generation

\begin{tabular}{|l|c|c|c|}
\hline $\mathbf{n}_{\mathbf{1}}^{\prime}$ & Ours & {$[34$} & {$[35]$} \\
\hline $\mathbf{5}$ & $\mathbf{0 . 0 0 9 1}$ & 0.330 & 0.0089 \\
\hline $\mathbf{1 0}$ & $\mathbf{0 . 0 0 9 3}$ & 0.468 & 0.0085 \\
\hline $\mathbf{1 5}$ & $\mathbf{0 . 0 0 8 8}$ & 0.605 & 0.0096 \\
\hline $\mathbf{2 0}$ & $\mathbf{0 . 0 0 9 6}$ & 0.743 & 0.0091 \\
\hline $\mathbf{2 5}$ & $\mathbf{0 . 0 0 9 2}$ & 0.881 & 0.0095 \\
\hline $\mathbf{3 0}$ & $\mathbf{0 . 0 0 9 1}$ & 1.018 & 0.0092 \\
\hline
\end{tabular}

Fig. 17 and Table 15 show the computation cost of keyword match test and the value of $\mathrm{x}$-axis varies with $l_{1}$. To simplify the comparison, the matrix row number $n_{1}$ is set to be 10 in scheme [35]. When $\left|l_{1}\right|=5$, the computation cost in schemes [34, 35] and our system are $0.180 \mathrm{~s}, 0.657 \mathrm{~s}$ and $0.0031 \mathrm{~s}$, respectively. When $\left|l_{1}\right|=30$, the computation cost in schemes [34, 35] and our system are $1.082 \mathrm{~s}, 2.009 \mathrm{~s}$ and $0.0034 \mathrm{~s}$, respectively. Our system has the least keyword match test cost. 


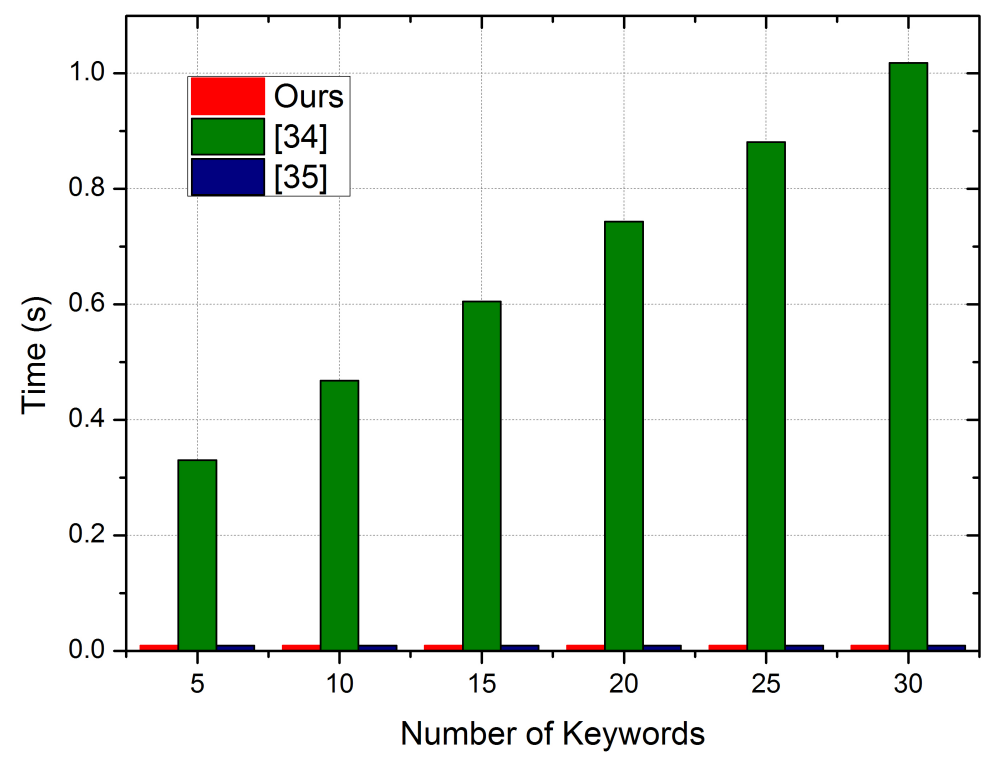

Figure 16: Computation Cost of Keyword Index Generation

Table 15: Computation Cost of Keyword Match Test

\begin{tabular}{|l|c|c|c|}
\hline $\mathbf{l}_{\mathbf{1}}$ & Ours & {$[34]$} & {$[35]$} \\
\hline $\mathbf{5}$ & $\mathbf{0 . 0 0 3 1}$ & 0.180 & 0.657 \\
\hline $\mathbf{1 0}$ & $\mathbf{0 . 0 0 2 9}$ & 0.361 & 0.927 \\
\hline $\mathbf{1 5}$ & $\mathbf{0 . 0 0 3 3}$ & 0.541 & 1.198 \\
\hline $\mathbf{2 0}$ & $\mathbf{0 . 0 0 3}$ & 0.721 & 1.468 \\
\hline $\mathbf{2 5}$ & $\mathbf{0 . 0 0 3 1}$ & 0.901 & 1.738 \\
\hline $\mathbf{3 0}$ & $\mathbf{0 . 0 0 3 4}$ & 1.082 & 2.009 \\
\hline
\end{tabular}




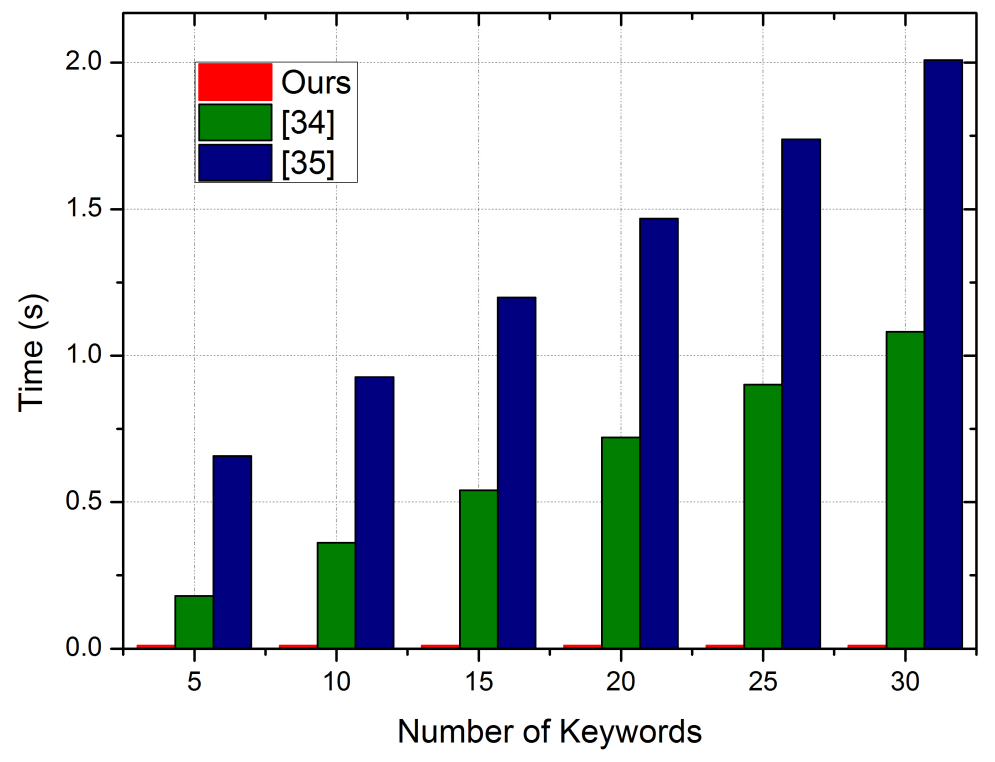

Figure 17: Computation Cost of Keyword Match Test 
Fig. 18 shows the computation cost comparison of the non-batch verification algorithm (Algorithm 8) and the batch verification algorithm (Algorithm 9. The value of x-axis in Fig. 18 varies with $\tau$ (the number of IoT ciphertexts). When $\tau=20$, the computation cost in non-batch and batch verification algorithms are $1.089 \mathrm{~s}$ and $0.588 \mathrm{~s}$, respectively. When $\tau=100$, the computation cost in non-batch and batch verification algorithms are $5.445 \mathrm{~s}$ and $2.795 \mathrm{~s}$, respectively. Compared with the non-batch verification algorithm, it can be seen that the batch verification algorithm is effective to reduce the computation cost of the patient device.

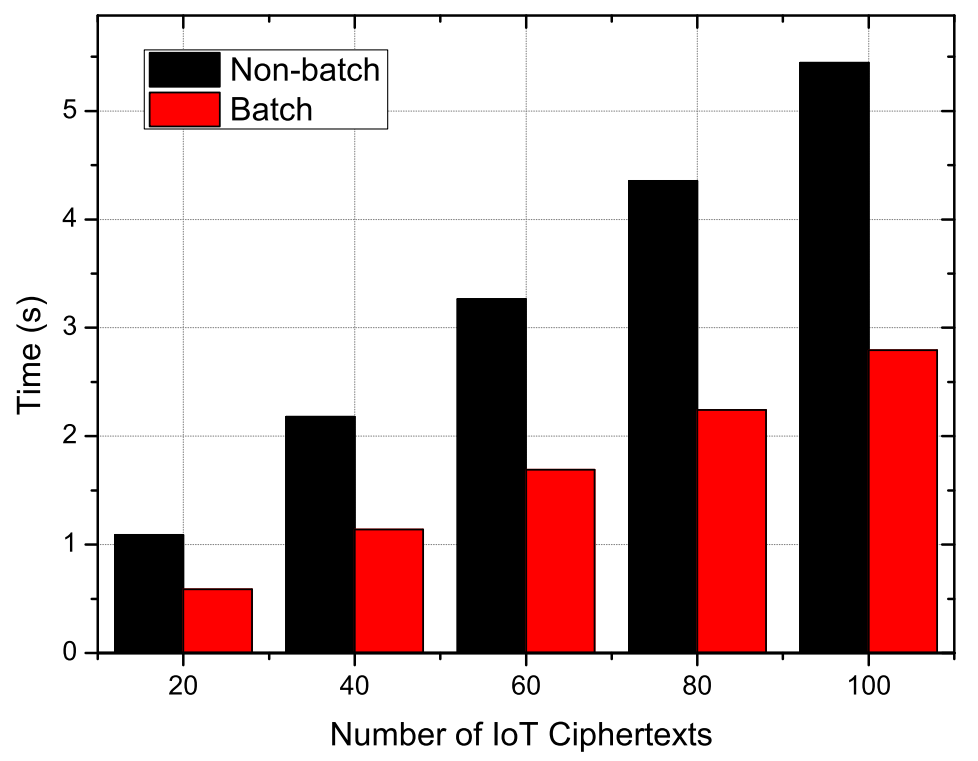

Figure 18: Computation Cost of Batch Verification

\section{Conclusion}

In this work, we have investigated the privacy-preserving fusion of IoT and big data in the e-health application scenario, and constructed a system to realize secure IoT communication and confidential medical big data storage. The system architecture and security model are defined for the proposed system. A 
non-interactive and authenticated key distribution procedure is designed for the medical IoT network. A batch authenticated verification algorithm is proposed to verify the source of encrypted IoT messages. Patients' EHRs are encrypted using the ABE methodology to realize access control. We also construct a novel keyword match based access policy updating mechanism to realize fine-grained policy updating control. We compare this system with other schemes and experiments are conducted to evaluate their performances. The testing results demonstrate that this system has performance outperforming the others and applicable in the e-health environment.

\section{Acknowledgement}

This work is supported by National Natural Science Foundation of China (No. 61402112, 61672159, 61702105); Open Fund Project of Fujian Provincial Key Laboratory of Information Processing and Intelligent Control (Minjiang University) (No. MJUKF201734); Fujian Major Project of Regional Industry (No. 2014H4015); Major Science and Technology Project of Fujian Province

(No. 2015H6013); Technology Innovation Platform Project of Fujian Province (No. 2014H2005), Fujian Collaborative Innovation Center for Big Data Application in Governments, Fujian Engineering Research Center of Big Data Analysis and Processing.

\section{References}

[1] Liao D, Sun G, Li H, Yu H, Chang V. The framework and algorithm for preserving user trajectory while using location-based services in IoT-cloud systems[J]. Cluster Computing, 2017, 20(3): 2283-2297.

[2] Sun G, Liao D, Li H, Yu H. Chang V. L2P2: A location-label based approach for privacy preserving in LBS[J]. Future Generation Computer Systems, 2017, 74: 375-384. 
[3] Yang Y, Zheng X, Liu X, et al. Cross-domain dynamic anonymous authenticated group key management with symptom-matching for e-health social system[J]. Future Generation Computer Systems, 2017.

[4] Chang V. The big data analysis for measuring popularity in the mobile cloud[J]. 2014.

[5] Sun G, Chang V, Ramachandran M, Sun Z, Li G, Yu H, Liao D. Efficient location privacy algorithm for Internet of Things (IoT) services and applications[J]. Journal of Network and Computer Applications, 2017, 89: $3-13$.

[6] Sun G, Xie Y, Liao D, Yu H, Chang V. User-defined privacy locationsharing system in mobile online social networks[J]. Journal of Network and Computer Applications, 2017, 86: 34-45.

[7] Arias O, Wurm J, Hoang K, et al. Privacy and security in internet of things and wearable devices[J]. IEEE Transactions on Multi-Scale Computing Systems, 2015, 1(2): 99-109.

[8] Daz-Snchez D, Sherratt R S, Almenarez F, et al. Secure store and forward proxy for dynamic IoT applications over M2M networks[J]. IEEE Transactions on Consumer Electronics, 2016, 62(4): 389-397.

[9] Li N, Liu D, Nepal S. Lightweight Mutual Authentication for IoT and Its Applications[J]. IEEE Transactions on Sustainable Computing, 2017.

[10] Zhang L, Zhang Y, Tang S, et al. Privacy Protection for E-health Systems by Means of Dynamic Authentication and Three-factor Key Agreement[J]. IEEE Transactions on Industrial Electronics, 2017.

[11] Wu F, Xu L, Kumari S, et al. An efficient authentication and key agreement scheme for multi-gateway wireless sensor networks in IoT deployment[J]. Journal of Network and Computer Applications, 2017, 89: 72-85. 
[12] Liu C, Chen J, Yang L T, et al. Authorized public auditing of dynamic big data storage on cloud with efficient verifiable fine-grained updates[J]. IEEE Transactions on Parallel and Distributed Systems, 2014, 25(9): 2234-2244.

[13] Baek J, Vu Q H, Liu J K, et al. A secure cloud computing based framework for big data information management of smart grid[J]. IEEE transactions on cloud computing, 2015, 3(2): 233-244.

[14] Liu C, Ranjan R, Yang C, et al. MuR-DPA: Top-down levelled multi-replica merkle hash tree based secure public auditing for dynamic big data storage on cloud[J]. IEEE Transactions on Computers, 2015, 64(9): 2609-2622.

[15] Yan Z, Ding W, Yu X, et al. Deduplication on encrypted big data in cloud[J]. IEEE transactions on big data, 2016, 2(2): 138-150.

[16] Boneh D, Di Crescenzo G, Ostrovsky R, et al. Public key encryption with keyword search[C]//Eurocrypt. 2004, 3027: 506-522.

[17] Xu P, Jin H, Wu Q, et al. Public-key encryption with fuzzy keyword search: A provably secure scheme under keyword guessing attack[J]. IEEE Transactions on computers, 2013, 62(11): 2266-2277.

[18] Yang Y, Zheng X, Chang V, et al. Semantic keyword searchable proxy re-encryption for postquantum secure cloud storage[J]. Concurrency and Computation: Practice and Experience, 2017, 29(19).

[19] Wang C, Cao N, Ren K, et al. Enabling secure and efficient ranked keyword search over outsourced cloud data[J]. IEEE Transactions on parallel and distributed systems, 2012, 23(8): 1467-1479.

[20] Cao N, Wang C, Li M, et al. Privacy-preserving multi-keyword ranked search over encrypted cloud data[J]. IEEE Transactions on parallel and distributed systems, 2014, 25(1): 222-233.

[21] Cash D, Jaeger J, Jarecki S, et al. Dynamic Searchable Encryption in VeryLarge Databases: Data Structures and Implementation[C]//NDSS. 2014, 14: $23-26$. 
[22] Li H, Liu D, Dai Y, et al. Engineering searchable encryption of mobile cloud networks: When QoE meets QoP[J]. IEEE Wireless Communications, 2015, 22(4): $74-80$.

[23] Yang Y, Ma M. Conjunctive keyword search with designated tester and timing enabled proxy re-encryption function for e-health clouds[J]. IEEE Transactions on Information Forensics and Security, 2016, 11(4): 746-759.

[24] Yang Y, Liu X, Deng R H, et al. Flexible Wildcard Searchable Encryption System[J]. IEEE Transactions on Services Computing, 2017.

[25] Goyal V, Pandey O, Sahai A, et al. Attribute-based encryption for finegrained access control of encrypted data $[\mathrm{C}] / /$ Proceedings of the 13th ACM conference on Computer and communications security. ACM, 2006: 89-98.

[26] Bethencourt J, Sahai A, Waters B. Ciphertext-policy attribute-based encryption $[\mathrm{C}] / /$ Security and Privacy, 2007. SP'07. IEEE Symposium on. IEEE, 2007: 321-334.

[27] Waters B. Ciphertext-policy attribute-based encryption: An expressive, efficient, and provably secure realization $[\mathrm{C}] / /$ Public Key Cryptography. 2011, 6571: 53-70.

[28] Ostrovsky R, Sahai A, Waters B. Attribute-based encryption with nonmonotonic access structures $[\mathrm{C}] / /$ Proceedings of the 14th ACM conference on Computer and communications security. ACM, 2007: 195-203.

[29] Yang Y, Liu X, Deng R H, et al. Lightweight Sharable and Traceable Secure Mobile Health System[J]. IEEE Transactions on Dependable and Secure Computing, 2017.

[30] Yang Y, Liu X, Deng R H. Lightweight Break-glass Access Control System for Healthcare Internet-of-Things[J]. IEEE Transactions on Industrial Informatics, 2017. 
[31] Yang K, Jia X, Ren K. Secure and verifiable policy update outsourcing for big data access control in the cloud[J]. IEEE Transactions on parallel and distributed systems, 2015, 26(12): 3461-3470.

[32] Li H, Liu D, Alharbi K, et al. Enabling fine-grained access control with efficient attribute revocation and policy updating in smart grid[J]. KSII Transactions on Internet and Information Systems (TIIS), 2015, 9(4): 14041423.

[33] Ying Z, Li H, Ma J, et al. Adaptively secure ciphertext-policy attributebased encryption with dynamic policy updating $[J]$. Science China Information Sciences, 2016, 59(4): 042701.

[34] Li J, Lin X, Zhang Y, et al. KSF-OABE: outsourced attribute-based encryption with keyword search function for cloud storage[J]. IEEE Transactions on Services Computing, 2017, 10(5): 715-725.

[35] Miao Y, Ma J, Liu X, et al. Attribute-Based Keyword Search over Hierarchical Data in Cloud Computing[J]. IEEE Transactions on Services Computing, 2017.

[36] Yeh L Y, Chiang P Y, Tsai Y L, et al. Cloud-based fine-grained health information access control framework for lightweight iot devices with dynamic auditing and attribute revocation[J]. IEEE Transactions on Cloud Computing, 2015.

[37] Beimel A, Secure Schemes for Secret Sharing and Key Distribution, PhD thesis, Israel Institute of Technology, Technion, Haifa, Israel, 1996.

[38] Lynn B. The Stanford Pairing Based Crypto Library. [Online]. Available: http://crypto.stanford.edu/pbc, accessed May 7, 2014. 